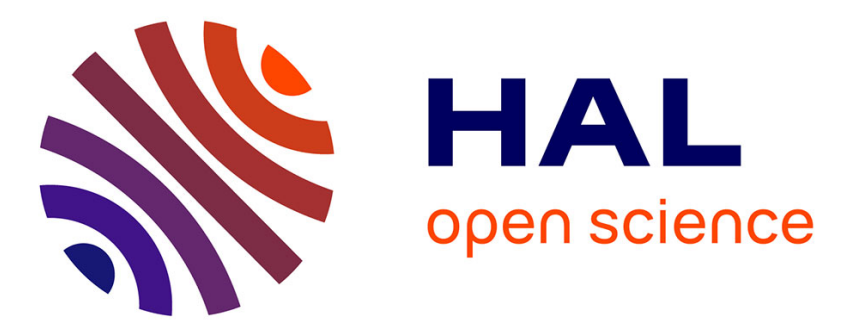

\title{
Corrélation entre freezing et dimension du noyau pédonculo-pontin dans la maladie de Parkinson: analyse semi-quantitative basée sur l'IRM cérébrale avec séquence FGATIR modifiée \\ Simone Chen
}

\section{To cite this version:}

Simone Chen. Corrélation entre freezing et dimension du noyau pédonculo-pontin dans la maladie de Parkinson: analyse semi-quantitative basée sur l'IRM cérébrale avec séquence FGATIR modifiée. Médecine humaine et pathologie. 2015. dumas-01289727

\section{HAL Id: dumas-01289727 https://dumas.ccsd.cnrs.fr/dumas-01289727}

Submitted on 17 Mar 2016

HAL is a multi-disciplinary open access archive for the deposit and dissemination of scientific research documents, whether they are published or not. The documents may come from teaching and research institutions in France or abroad, or from public or private research centers.
L'archive ouverte pluridisciplinaire HAL, est destinée au dépôt et à la diffusion de documents scientifiques de niveau recherche, publiés ou non, émanant des établissements d'enseignement et de recherche français ou étrangers, des laboratoires publics ou privés. 
Université de Picardie - Jules Verne

UFR de Médecine d'Amiens

Année 2015

Thèse $n^{\circ} 2015-90$

\section{CORRELATION ENTRE FREEZING ET DIMENSION DU NOYAU PEDONCULO-PONTIN DANS LA MALADIE DE PARKINSON : ANALYSE SEMI-QUANTITATIVE BASEE SUR L'IRM CEREBRALE AVEC SEQUENCE FGATIR MODIFIEE}

Directeur de thèse : Madame le Docteur Mélissa TIR

Thèse pour le diplôme d'Etat de Docteur en Médecine

Neurologie

Présentée et soutenue publiquement

Le 8 septembre 2015

Par

Simone CHEN

Membres du Jury:

Président du Jury : Monsieur le Professeur Godefroy

Assesseurs : $\quad$ Monsieur le Professeur Macron

Monsieur le Professeur Krystkowiak

Monsieur le Professeur Constans

Monsieur le Docteur Lefranc

Madame le Docteur Tir 
Monsieur le Professeur Olivier GODEFROY

Professeur des Universités-Praticien Hospitalier

(Neurologie)

Chef du Service de Neurologie

Pôle Autonomie

Monsieur le Professeur,

Je vous remercie d'avoir accepté de présider ce jury, d'avoir suscité mon intérêt pour la Neurologie, et de l'enseignement que vous avez pu m'apporter durant mon internat. 
Monsieur le Professeur Jean-Michel MACRON

Professeur des Universités-Praticien Hospitalier

(Physiologie)

Chef du Service Explorations Fonctionnelles du Système Nerveux

Pôle Autonomie

Monsieur le Professeur,

Je vous remercie d'avoir accepté de juger mon travail, et de l'enseignement que vous avez pu m'apporter lors de mes passages dans votre service. 
Monsieur le Professeur Pierre KRYSTKOWIAK

Professeur des Universités-Praticien Hospitalier

(Neurologie)

Assesseur du $2^{\text {ème }}$ cycle

Monsieur le Professeur,

Je vous remercie d'avoir dirigé mon travail en «back-up », comme vous le dites si bien. Vous avez contribué à susciter mon intérêt pour la Neurologie, notamment pour la maladie de Parkinson et la pathologie du mouvement. Merci de l'enseignement que vous m'avez apporté au cours de mon internat. 
Monsieur le Professeur Jean-Marc CONSTANS

Professeur des Universités-Praticien Hospitalier

(Radiologie et Imagerie médicale)

Pôle Imagerie

Monsieur le Professeur,

Je vous remercie d'avoir accepté d'être membre du jury de cette thèse. Veuillez trouver ici l'expression de mon sincère respect. 
Monsieur le Docteur Michel LEFRANC

Maître de Conférences des Universités - Praticien Hospitalier

(Neurochirurgie)

Michel,

Merci pour votre aide si précieuse, vos bons conseils et votre disponibilité dans l'élaboration de ce travail, qui n'aurait pu aboutir sans vous... même si vous étiez initialement «très inquiet»... Un grand MERCI pour tout! Je vous suis infiniment reconnaissante. 
Madame le Docteur Mélissa TIR

Praticien Hospitalier

(Neurologie)

Mélissa,

Je vous remercie d'avoir dirigé ma thèse, de m'avoir guidée tout au long de cette aventure.

L'enseignement que vous m'avez prodigué dans le service de Neurologie, notamment en matière de maladie de Parkinson et pathologie du mouvement, m'est très précieux. 


\section{REMERCIEMENTS}

A mes parents : c'est tout simplement grâce à vous si j'en suis là. Merci de votre aide, amour et soutien au quotidien.

A Arnaud, merci pour ton soutien depuis tant d'années et merci de me supporter au quotidien malgré mon sale caractère...

A Sylvain, mon «p'tit » frère, merci de m'avoir aidée à dompter Matlab, c'est grâce à toi si j'ai un magnifique schéma tridimensionnel ;-) et j'en suis très fière ! Merci d'être là pour moi tout simplement.

A Sébastien, mon «grand » frère, même si des centaines de kilomètres nous séparent, je sais que je peux compter sur toi, et grâce à toi, je fais de la «bobologie » à distance ;-)

A ma meilleure amie Chloé (et son David, sans oublier Pierre-Louis), tu es comme une sœur pour moi. Merci de m'avoir soutenue durant tant d'années.

A Julie, quelles superbes retrouvailles après tant d'années. Nos souvenirs d'enfance restent intacts. A très bientôt pour une nouvelle soirée parisienne ;-)

A mes copines : Marion, Camille, Clém, Crevette, Cécile, Laure, Myriam, Marion L, nos chemins se sont séparés depuis l'ECN, mais je ne vous oublie pas.

A mes chefs :

Bertille et Philippe, apprendre à vos côtés fut un réel plaisir, merci pour tout ce que vous m'avez apporté. Des mots et des phrases cultes restent gravés dans ma mémoire ;-) J'attends maintenant avec impatience la remise du stimulateur et du casque d'or ;-)

Audrey, d'abord co-interne puis chef, ce fut un bonheur de travailler avec toi ! Lucie, Céline, Mathieu : merci pour tout ce que vous m'avez appris.

A mes co-internes :

Anaïs, Mélanie et Yassine, qui ont vécu la même galère et qui sont de véritables amis. Julie et son blot par blot qui est devenue ma sœurette de neurologie générale ;-) 
A toute l'équipe paramédicale de Neurologie du CHU d'Amiens, notamment Meggy, Béa Schu, Françoise...

A toute l'équipe paramédicale des EFSN : Delphine, les Pascale (PL1 et PL2), Flo, Hélène, Caro, Corinne, Fabien : merci pour votre bonne humeur et vos petits repas de service.

A M. Bouffeteau, je vous remercie de l'attention que vous m'accordez en m'ouvrant les portes de votre service.

A toute l'équipe paramédicale de Neurologie de Saint Quentin : notamment Isa alias Mme Formidable, Lulu, Stéph, Tam, Vincent, Véro...

A toute l'équipe médicale de Neurologie et MPR de Compiègne : travailler à vos côtés fut un vrai plaisir. 


\section{TABLES DES MATIERES}

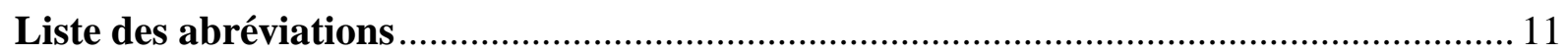

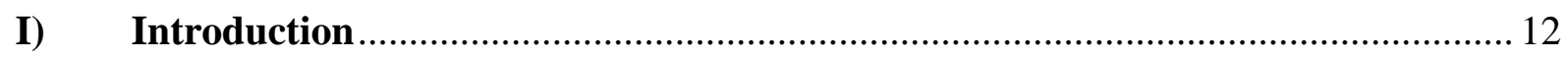

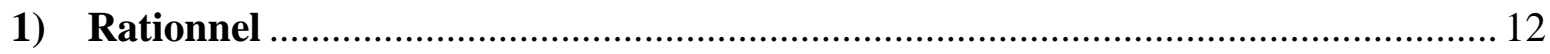

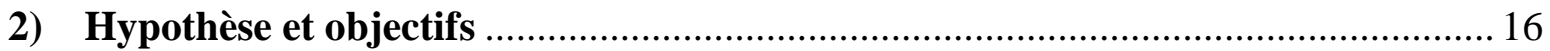

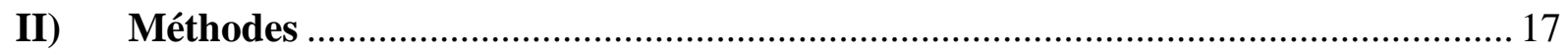

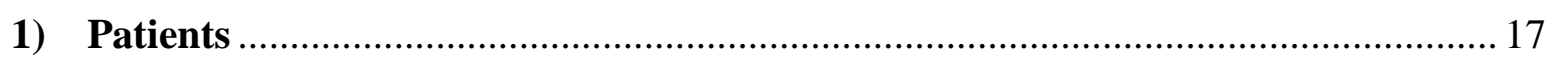

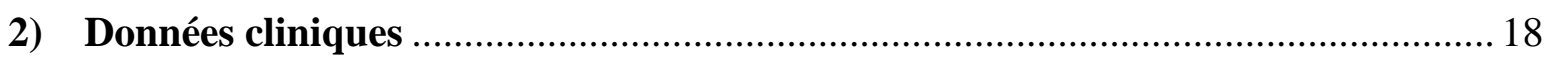

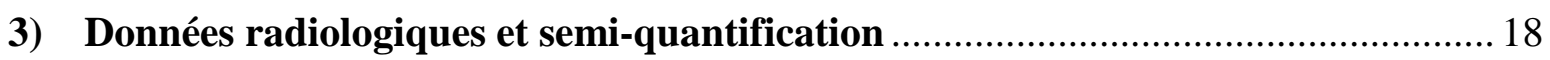

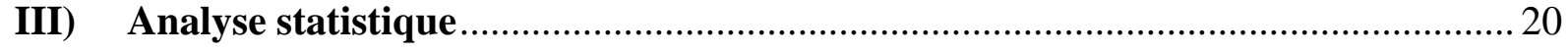

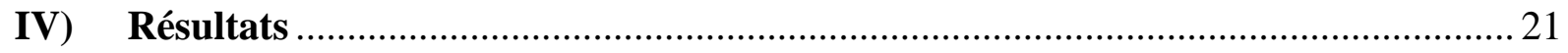

1) Caractéristiques cliniques des différents groupes ............................................ 21

2) Coordonnées stéréotaxiques barycentriques des PPN ...................................... 22

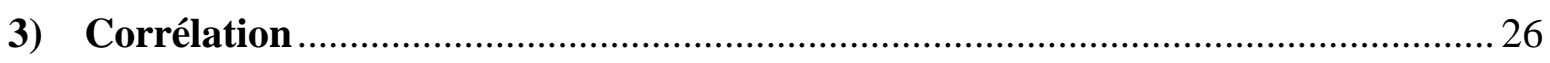

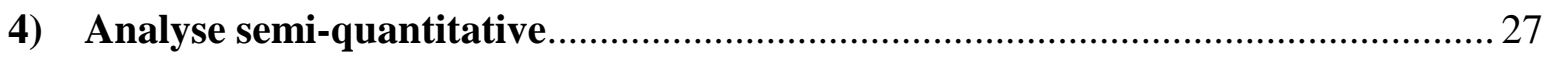

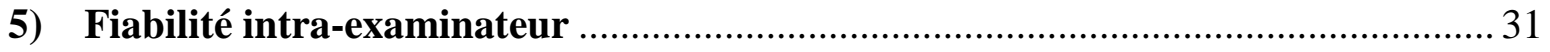

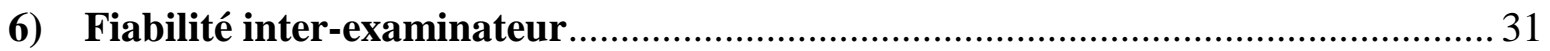

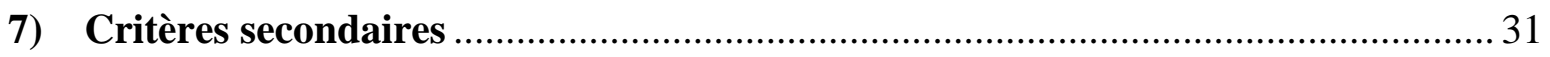

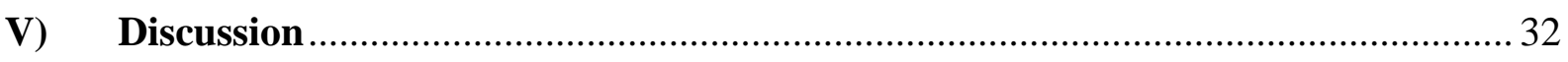

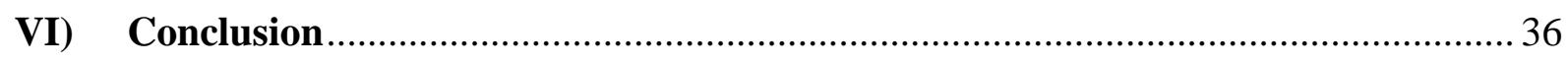

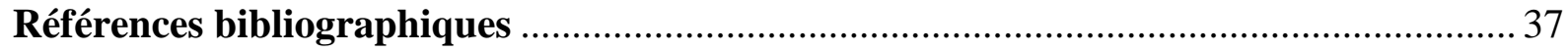




\section{$\underline{\text { Liste des abréviations }}$}

CA : commissure antérieure

$\mathrm{CP}$ : commissure postérieure

DRS : Dementia Rating Scale

FGATIR : Fast Gray Matter Acquisition T1 Inversion Recovery

FOG : freezing of gait

IRM : imagerie par résonance magnétique

LARS : Lille Apathy Rating Scale

MADRS : Montgomery and Asberg Depression Rating Scale

MP : maladie de Parkinson

NST : noyau sous thalamique

PPN : noyau pédonculo-pontin

PPNc : pars compacta du noyau pédonculo-pontin

PPNd : pars dissipatus du noyau pédonculo-pontin

SCP : stimulation cérébrale profonde

SPGR : Spoiled Gradient Recalled Echo

UPDRS : Unified Parkinson Disease Rating Scale 


\section{I) Introduction}

\section{1) Rationnel}

La maladie de Parkinson (MP) est fréquente et représente 1,7\% de la population européenne après 65 ans [1]. Il s'agit d'une maladie neurodégénérative principalement caractérisée par des troubles moteurs qui constituent une triade associant akinésie, tremblement de repos et rigidité. Cette triade est principalement liée à un dysfonctionnement du système des ganglions de la base qui est lui-même la conséquence d'une dégénérescence de la voie dopaminergique nigro-striée, en l'occurrence les neurones dopaminergiques de la pars compacta de la substance noire. Les troubles de la marche, qui sont souvent associés à cette triade peuvent s'observer à la phase précoce de la maladie. Ils se caractérisent par une vitesse diminuée, une réduction de la longueur du pas, une augmentation de la durée de la phase d'appui bipodal et une diminution du ballant des bras. Au cours de l'évolution de la maladie, les troubles de la marche s'aggravent, avec l'apparition de festinations et d'un freezing et s'intègrent dans ce que l'on appelle les signes moteurs axiaux tardifs au même titre que la dysarthrie, les troubles de la déglutition et l'instabilité posturale. Les festinations correspondent à une brusque augmentation de fréquence et diminution d'amplitude de la marche donnant l'impression que le patient court après son centre de gravité en accélérant le pas. Le freezing (ou freezing of gait «FOG») est défini comme une akinésie paroxystique ou enrayage cinétique de la marche : le patient décrit la sensation d'avoir les pieds collés au sol, de faire des petits pas sur place. Il survient notamment au démarrage, au demi-tour, lors du passage d'une porte et dans des situations où l'attention du patient est détournée. Au cours de l'évolution de la MP, la prévalence du freezing augmente et il est observé chez $52 \%$ des patients après 8 ans d'évolution [2]. Il constitue un problème majeur car il peut être responsable de chutes et d'une perte d'autonomie significative entravant alors la qualité de vie, sans oublier son association fréquente à un syndrome dépressif [3-4]. La présence d'un freezing est associée à la sévérité des signes axiaux, la sévérité de la maladie (score de Hoehn et Yahr), la durée d'évolution de la maladie, la présence d'une dysarthrie, la présence d'une démence sous corticale et/ou d'un syndrome dysexécutif et la durée de la dopathérapie [5-6]. Le freezing peut cependant apparaitre beaucoup plus précocement, dès la deuxième année d'évolution dans $7 \%$ des cas [7]. A ce stade, il est sensible à la dopathérapie (freezing dopa-sensible) alors qu'à un stade plus avancé de la maladie, il devient volontiers dopa-résistant. Cela s'explique par 
l'implication progressive, au fil de l'évolution de la maladie, de lésions des systèmes non dopaminergiques sur lesquelles les traitements dopaminergiques habituels sont sans effet.

Parmi l'atteinte de ces systèmes non dopaminergiques, plusieurs études ont montré que les neurones cholinergiques du noyau pédonculo-pontin (PPN) dégénéraient dans la MP et étaient impliqués dans la physiopathologie du freezing [8-10].

Le PPN est une structure mésopontique constituée d'une population neuronale hétérogène, située sous le noyau rouge et dorsalement à la substance noire. La Figure 1 illustre la localisation du PPN. Il est classiquement scindé en deux parties en fonction de la taille et de la densité de neurones qu'elles contiennent: la pars compacta (PPNc) et la pars dissipatus (PPNd) [11]. Le PPNc est situé dans la moitié caudale et la partie dorsolatérale du noyau, il est constitué essentiellement de grandes cellules cholinergiques. Le PPNd est situé autour de l'axe rostro-caudal du noyau et est constitué de neurones de petite et moyenne tailles, cholinergiques, glutamatergiques et gabaergiques [12].

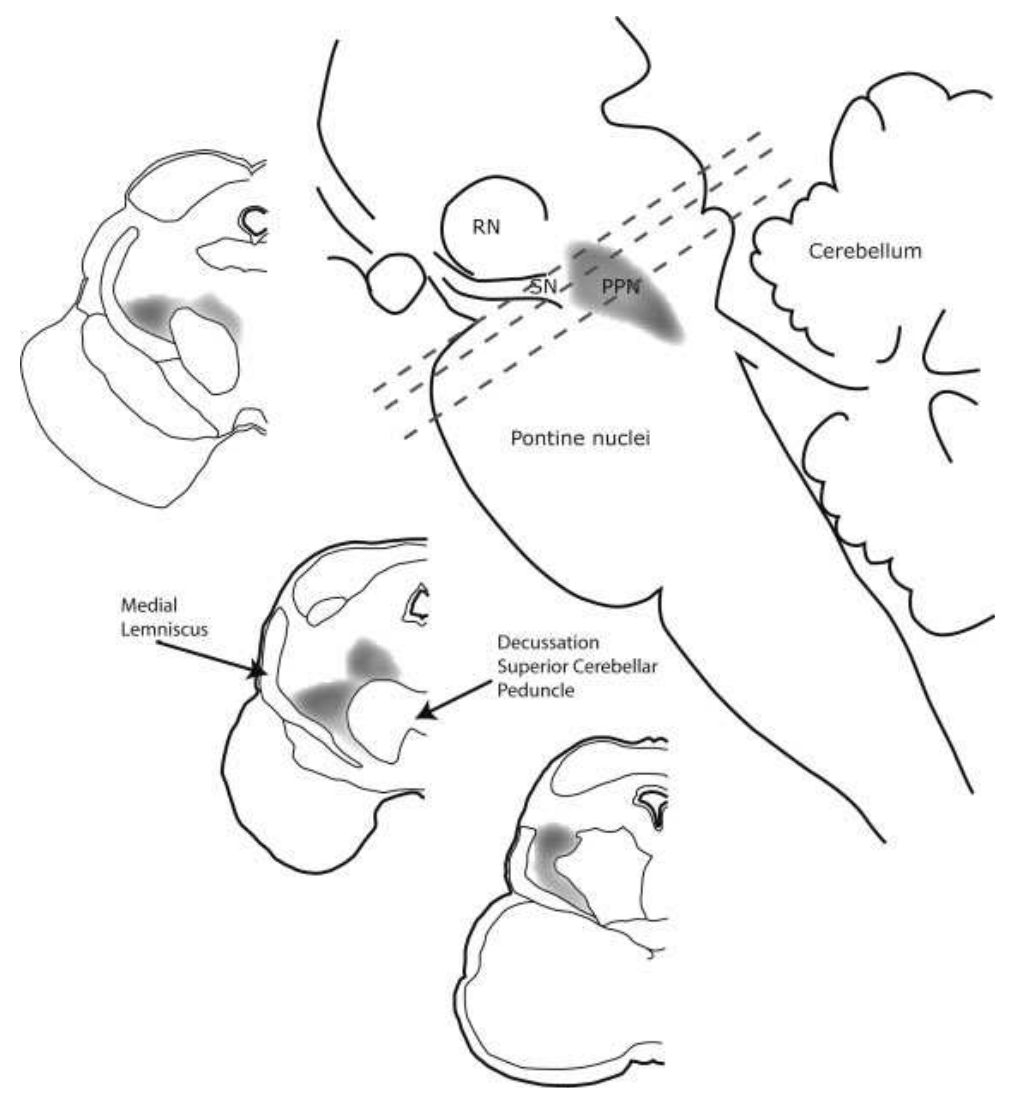

Figure 1: Coupes axiales montrant la localisation du PPN (Jenkinson, 2009) 
Le PPN est connecté à l'ensemble des structures clés du contrôle moteur. La Figure 2 illustre ses principales connexions. Il reçoit des projections directes du cortex moteur, est relié aux ganglions de la base, projette sur le thalamus, les aires motrices du tronc cérébral et la moelle épinière. A travers ces connexions, le PPN est impliqué dans le mécanisme de veille, le contrôle comportemental et locomoteur et le tonus musculaire.

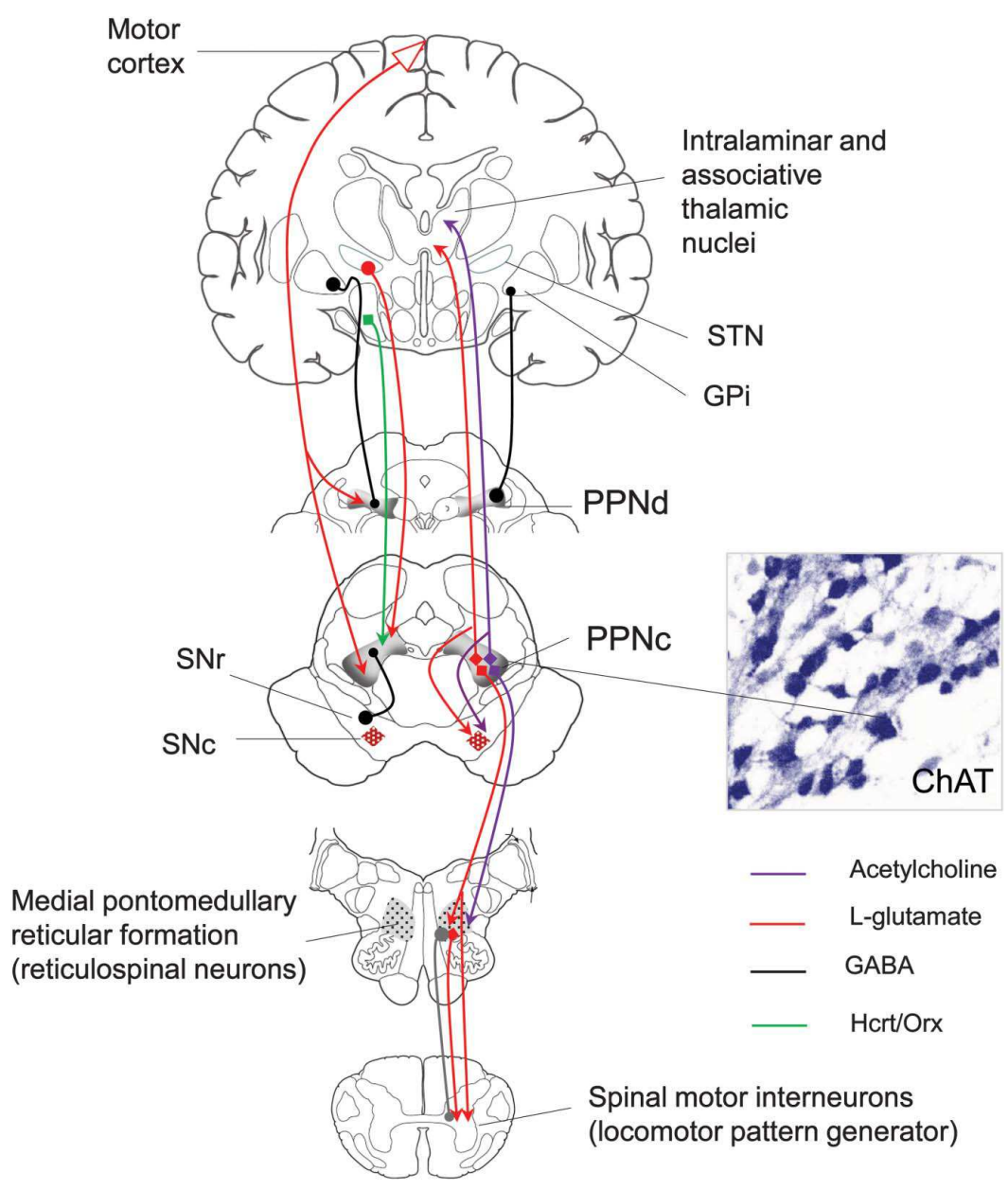

Figure 2: Principales connexions du PPN (Benarroch, 2013)

STN: noyau sous thalamique, GPi: globus pallidum interne, PPNd: pars dissipatus du noyau pédonculo-pontin, PPNc : pars compacta du noyau pédonculo-pontin, SNr : substance noire réticulée, SNc : substance noire compacte, ChAT : choline acétyltransférase

Dans l'étude de Karachi et collaborateurs portant sur des modèles animaux [13], il a été mis en évidence qu'une lésion spécifique du PPN entrainait des troubles axiaux (troubles de la marche et posturaux) ne répondant pas aux médications dopaminergiques. Dans cette même étude, une quantification du nombre de neurones cholinergiques a été réalisée dans trois 
groupes de cerveaux humains (groupe de sujets sains, groupe de patients parkinsoniens sans troubles de la marche ni chutes et groupe de patients parkinsoniens avec troubles de la marche et chutes): on retrouvait dans le groupe des parkinsoniens «chuteurs » un nombre de neurones cholinergiques significativement plus bas que dans les autres groupes [13].

A la suite de ce type d'études mettant en exergue le rôle du PPN dans la physiopathologie du freezing, plusieurs équipes, depuis une dizaine d'années, ont choisi comme cible de stimulation cérébrale profonde (SCP) le PPN [14-20], avec une stimulation à basse fréquence du fait d'effets indésirables invalidants lorsque la fréquence de stimulation était plus haute. Les patients sélectionnés étaient des parkinsoniens avec des troubles de la marche doparésistants ayant auparavant bénéficié ou non d'une stimulation des noyaux sous-thalamiques (NST). Les résultats qui découlent de ces études sont hétérogènes. Initialement, les résultats des études réalisées en ouvert [14-16] étaient très encourageants montrant une efficacité de la SCP du PPN sur les troubles de la marche. Cependant, cela n'a pas été confirmé par une étude en double aveugle [17]. Toutefois, Thevathasan et collaborateurs [20] ont récemment montré de manière objective une amélioration du freezing après stimulation du PPN. La grande variabilité des résultats issus de ces études est probablement en lien avec plusieurs paramètres : une population cible hétérogène, des critères cliniques difficiles à quantifier du fait de leur caractère paroxystique et survenant dans des conditions particulières (freezing, chutes), et bien entendu la difficulté à cibler le PPN de manière précise.

En effet, le PPN est une structure anatomique mal délimitée dans les atlas anatomiques de référence (notamment l'atlas stéréotaxique de Schaltenbrand et Wahren [21]). Zrinzo et al. [22] ont pu établir en 2008 des coordonnées du PPN à partir des imageries par résonance magnétique (IRM) préopératoires de 12 patients éligibles à une SCP en s'aidant des informations couplées de deux atlas : celui d'Afshar [23] et celui de Paxinos et Huang [24]. Une séquence pondérée $\mathrm{T} 1$ a été réalisée en utilisant une séquence axiale volumétrique orthogonale Spoiled Gradient Recalled Echo (SPGR). La moyenne des coordonnées stéréotaxiques barycentriques issues de sa population de patients correspond aux coordonnées suivantes : le PPN rostral est situé $6 \mathrm{~mm}( \pm 0,5)$ en latéral et $3 \mathrm{~mm}( \pm 1,1)$ en arrière de la commissure postérieure $(\mathrm{CP})$ et $9 \mathrm{~mm}( \pm 1,1)$ sous l'axe commissure antérieure - commissure postérieure (CA-CP), le PPN caudal est situé $6,8 \mathrm{~mm}( \pm 0,5)$ en latéral, $4 \mathrm{~mm}( \pm 1,1)$ en arrière de $\mathrm{CP}$ et $13,9 \mathrm{~mm}( \pm 1,2)$ sous l'axe CA-CP. Il s'agit à notre connaissance de la seule étude ayant permis de recueillir des coordonnées précises du PPN en utilisant une séquence IRM chez des patients vivants. Ces coordonnées, couplées aux données des atlas anatomiques, sont fréquemment utilisées par les neurochirurgiens qui envisagent une SCP du PPN. 
Il est bien admis que certains patients ayant bénéficié d'une SCP des NST peuvent développer par la suite des troubles de la marche dopa-résistants, particulièrement un freezing même si les patients sont en général rigoureusement sélectionnés pour n'avoir aucun (sinon des signes minimes) trouble de la marche et/ou instabilité posturale en best On lors du bilan d'éligibilité préopératoire. Cependant, certains de ces patients peuvent parfois présenter un freezing en worst Off qui doit être totalement dopa-sensible si l'on veut proposer ce type de chirurgie au patient.

Actuellement, nous sommes incapables de prédire le risque pour un patient donné, traité par SCP des NST, de développer un freezing, son caractère potentiellement dopa-sensible et le délai avec lequel il risque d'apparaître après la chirurgie. L'idée que nous pourrions commencer à y parvenir a émergé avec la mise au point d'une séquence 3D T1 inversion récupération avec destruction de l'aimantation résiduelle remaniée, séquence communément appelée Fast Gray Matter Acquisition T1 Inversion Recovery (FGATIR). Cette séquence a initialement été mise au point pour le repérage des pallidums internes pour la SCP des patients souffrant de pathologies dystoniques. C'est à cette occasion que le neurochirurgien responsable de l'activité de SCP du CHU d'Amiens et le neuroradiologue se sont aperçus que le PPN était également très bien visualisé sur cette séquence FGATIR modifiée.

\section{2) Hypothèse et objectifs}

Avant d'envisager une étude longitudinale, il convenait de débuter par une étude cas-témoin. Notre hypothèse de base est que les patients parkinsoniens présentant un freezing ont un PPN plus petit que les patients parkinsoniens ne présentant pas de freezing, ainsi que cela a été suggéré par les études portant sur les modèles animaux [13].

L'objectif principal de cette étude est ainsi de montrer, au moyen d'une évaluation semiquantitative en IRM, que le freezing dans la MP est associé à un PPN de taille réduite.

Les objectifs secondaires sont de montrer une corrélation entre d'une part la taille du PPN, et d'autre part différents scores cliniques tels que le sous score axial (score composite issu de la partie motrice de l'échelle Unified Parkinson Disease Rating Scale, UPDRS III [25]), ainsi que les données cognitives et psychocomportementales (dépression et apathie). 


\section{II) Méthodes}

\section{1) Patients}

Il s'agit d'une étude observationnelle rétrospective cas-témoin dans laquelle ont été inclus de manière consécutive tous les patients éligibles à une SCP au Centre Hospitalier Universitaire (CHU) d'Amiens de mai 2011 à octobre 2014. Trois groupes ont été formés : le premier groupe comporte les patients parkinsoniens présentant un freezing de la marche en préopératoire $(\mathrm{FOG}+)$, le deuxième les patients parkinsoniens ne présentant pas de freezing en préopératoire (FOG -) et le troisième groupe correspond au groupe contrôle constitué de patients éligibles à une SCP pour une pathologie autre qu'une MP et dont la neuropathologie épargne le PPN. La catégorisation entre les groupes FOG + et FOG - a été réalisée de manière binaire : présence ou non d'un freezing en préopératoire, sans intervention de la sévérité.

Les critères d'inclusion des patients parkinsoniens freezers $(F O G+)$ et non freezers (FOG -) étaient les suivants : âge supérieur à 18 ans, patient coopérant et capable de donner son consentement, MP remplissant les critères d'éligibilité à une SCP des noyaux sous thalamiques (NST) [26] : évolution de la maladie depuis au moins 5 ans, stade des fluctuations motrices et dyskinésies ou grand tremblement non contrôlé par le traitement médicamenteux, dopa-sensibilité supérieure ou égale à 33\%, absence de troubles cognitifs représenté par un score obtenu à la Dementia Rating Scale (DRS) de Mattis [27] supérieur ou égal 130/144.

Les critères d'inclusion du groupe contrôle étaient les suivants : âge supérieur à 18 ans, patient coopérant et capable de donner son consentement, éligible à une SCP dans le cadre d'une pathologie neurologique autre que la MP et sans dégénérescence du PPN.

L'ensemble des patients devait avoir bénéficié en préopératoire d'une IRM encéphalique incluant une séquence FGATIR modifiée.

Les critères de non inclusion des groupes FOG + et FOG - étaient : l'incapacité à donner son consentement, handicap moteur limité apprécié par le score moteur de l'échelle UPDRS en worst Off (inférieur à 30) sauf dans les formes tremblantes pures et les formes sévères, pathologie intercurrente (cardiaque, respiratoire...), mauvaise adhésion au traitement médical, suivi irrégulier, faible dopa-sensibilité, contre-indication à la réalisation de l'IRM préopératoire, persistance de signes axiaux marqués en best On, score obtenu à la DRS de Mattis inférieur à 130. 
Les critères de non-inclusion du groupe contrôle étaient: l'incapacité à donner son consentement, pathologie intercurrente (cardiaque, respiratoire....), mauvaise adhésion au traitement médical, suivi irrégulier, contre-indication à la réalisation de l'IRM préopératoire.

\section{2) Données cliniques}

Nous avons recueilli de manière rétrospective les données démographiques de chaque patient (âge, sexe) ainsi que les caractéristiques de la MP pour les groupes FOG + et FOG - : durée d'évolution de la maladie, score moteur (partie III) de la Unified Parkinson's Disease Rating Scale (UPDRS III) en condition worst Off (à jeun de traitement antiparkinsonien) et en condition best On (après administration aigue d'une dose supraliminaire de Levodopa [28]), sous score axial composite de l'UPDRS III comportant les items 18 : parole, 27 : lever, 28 : posture, 29: stabilité, 30 : démarche, en conditions worst Off et best On, données du bilan neuropsychologique comportant les scores aux échelles DRS de Mattis, d'évaluation de la dépression de Montgomery et Asberg (Montgomery and Asberg Depression Rating Scale : MADRS) [29], d'apathie de Lille (Lille Apathy Rating Scale : LARS) [30].

\section{3) Données radiologiques et semi-quantification}

L'ensemble des patients inclus a ainsi bénéficié d'une IRM encéphalique préopératoire sous anesthésie générale afin d'obtenir une parfaite résolution des structures anatomiques sans artéfacts de mouvements. Les acquisitions ont été réalisées sur une IRM 3 Teslas (SIGNA HDx MRHDX; General Electric Medical Systems). Parmi les séquences réalisées dans le cadre du repérage préopératoire, les patients ont bénéficié d'une séquence FGATIR modifiée (512×512, 1 mm d'épaisseur, en coupes jointives). Il s'agit d'une séquence 3D T1 inversion récupération avec destruction de l'aimantation résiduelle qui permet de visualiser avec une meilleure résolution et un meilleur contraste les structures anatomiques cibles de la SCP.

Cette séquence a été analysée par le logiciel ROSANNA (Medtech, Montpellier France), logiciel de stéréotaxie de neurochirurgie, avec un reformatage des images dans un plan parallèle à la ligne inter-commissurale, ligne allant de $\mathrm{CA}$ à $\mathrm{CP}$. Cette ligne $\mathrm{CA}-\mathrm{CP}$ a été réalisée pour chaque patient par le même intervenant expérimenté, neurochirurgien fonctionnel responsable de l'activité de stimulation cérébrale profonde du CHU d'Amiens (Dr Lefranc). Le choix d'un seul intervenant permet d'éviter une variabilité interindividuelle lors 
de la constitution de cette ligne, dont on sait qu'elle peut varier d'un intervenant à l'autre, variabilité pouvant atteindre jusqu’à 30\% [31-32].

Dans un premier temps, nous avons tout d'abord voulu confirmer que la structure visualisée sur notre séquence FGATIR modifiée correspondait réellement au PPN. Nous avons ainsi recueilli les coordonnées stéréotaxiques barycentriques tridimensionnelles IRM, en fonction de la ligne CA-CP, des présumés PPN de l'ensemble des patients inclus et les avons comparé aux coordonnées recueillies par Zrinzo et al. [22]

Dans un second temps, nous avons évalué de manière semi-quantitative le degré d'atrophie du PPN pour chaque patient, selon la classification suivante : $0=$ pas d'atrophie, $1=$ atrophie légère, 2 = atrophie modérée, 3 = atrophie sévère ou PPN non visible. Pour chaque patient, quatre mesures ont été réalisées : partie rostrale et partie caudale des PPN droit et gauche.

L'évaluation a été réalisée de façon randomisée, en aveugle à l'aide de la séquence IRM FGATIR modifiée en coupe axiale reformatée dans le plan CA-CP.

La fiabilité intra-examinateur a été évaluée par le neurologue (Dr Chen) après avoir réalisé les mesures des PPN de 10 patients consécutifs, de manière randomisée et en aveugle, à deux reprises. Les deux évaluations ont été réalisées à une semaine d'intervalle.

La fiabilité inter-examinateur a été évaluée de la même manière, en réalisant les mesures semi-quantitatives des PPN de 10 patients consécutifs, après randomisation et en aveugle, le premier examinateur était un neurologue (Dr Chen), le deuxième était un neurochirurgien (Dr Lefranc). 


\section{III) Analyse statistique}

Les données démographiques de l'ensemble des patients ont été comparées à l'aide du test exact de Fisher pour les variables qualitatives et par le test de Kruskall-Wallis pour les variables quantitatives. Les caractéristiques de la MP et les données du bilan neuropsychologique des groupes FOG + et FOG - ont été comparées à l'aide du test de Mann-Whitney pour les variables quantitatives. L'intervalle de confiance pour l'odds ratio était ajusté à $95 \%$.

Les coordonnées stéréotaxiques barycentriques des PPN ont été comparées à celles décrites par Zrinzo et al. grâce au test t de Student.

La corrélation entre les différentes parties des PPN droits et gauches a été évaluée par le test de Spearman.

Les fiabilités inter examinateur et intra examinateur ont été recherchées à l'aide du coefficient Kappa de Cohen.

L'évaluation du critère principal de jugement et des critères secondaires a été réalisée par le test de Kruskall-Wallis.

Le risque alpha était fixé à 5\% pour l'ensemble des analyses, sauf pour l'évaluation du critère principal de jugement et des critères secondaires où il était fixé à $1,25 \%$. Nous avions en effet quatre variables définissant ce critère, raison pour laquelle le risque alpha a été ajusté. 


\section{IV) Résultats}

\section{1) Caractéristiques cliniques des différents groupes}

Trente patients ont été inclus au CHU d'Amiens de mai 2011 à octobre 2014, de manière rétrospective, répartis en trois groupes. Le groupe FOG + était composé de 12 patients, le groupe FOG - comportait 13 patients. Le groupe contrôle était constitué de 5 sujets ayant bénéficié d'une stimulation cérébrale profonde dans le cadre d'une pathologie neurologique autre que la maladie de Parkinson (dystonie focale, localisée ou généralisée). Les données démographiques et médicales des patients sont représentées dans le Tableau 1.

\begin{tabular}{|l|c|c|c|c|}
\hline & FOG + & FOG - & Contrôles & p \\
\hline N & 12 & 13 & 5 & \\
\hline Sex Ratio (H/F) & $7 / 5$ & $8 / 5$ & $3 / 2$ & 1 \\
\hline Age (années) & $65,33( \pm 7,04)$ & $60,62( \pm 8,21)$ & $51,2( \pm 15,1)$ & 0,09 \\
\hline Durée d'évolution (années) & $12,58( \pm 4,03)$ & $11,3( \pm 3,28)$ & NA & 0,495 \\
\hline UPDRS III worst Off $(/ \mathbf{1 0 8})$ & $30,83( \pm 9)$ & $32,62( \pm 9,69)$ & NA & 0,683 \\
\hline UPDRS III best On $(/ \mathbf{1 0 8})$ & $13,75( \pm 6,06)$ & $15,15( \pm 6,27)$ & NA & 0,785 \\
\hline Dopa-sensibilité (\%) & $56,17( \pm 13,82)$ & $52,85( \pm 13,73)$ & NA & 0,355 \\
\hline Sous score axial worst Off $(/ \mathbf{2 0})$ & $6,58( \pm 2,02)$ & $5,25( \pm 2,86)$ & NA & 0,066 \\
\hline Sous score axial best On $(\mathbf{/ 2 0})$ & $3( \pm 2,04)$ & $2,83( \pm 2,08)$ & NA & 0,768 \\
\hline DRS de Mattis $(/ \mathbf{1 4 4})$ & $138,08( \pm 3,90)$ & $136,85( \pm 4,10)$ & NA & 0,249 \\
\hline MADRS $(/ 60)$ & $8,56( \pm 3,09)$ & $6,75( \pm 4,05)$ & NA & 0,299 \\
\hline LARS $(\mathbf{/ 3 6 )}$ & $-24,5( \pm 5,80)$ & $-25,63( \pm 6,37)$ & NA & 0,894 \\
\hline
\end{tabular}

Tableau 1: Caractéristiques cliniques des différents groupes

Moyennes ( \pm écart-types), $p$ : seuil de significativité <0,05.

UPDRS III : Unified Parkinson's Disease Rating Scale III, DRS : Dementia Rating Scale, MADRS : Montgomery and Asberg Depression Rating Scale, LARS : Lille Apathy Rating Scale, FOG +: freezer, FOG - : non freezer.

Les 3 groupes étaient comparables en sexe et âge (respectivement $\mathrm{p}=1$ et $\mathrm{p}=0,09$ ).

Les groupes FOG + et FOG - étaient comparables sur la durée d'évolution de la maladie, le score UPDRS III et sous score axial en conditions best On et worst Off, la dopa-sensibilité et les données du bilan neuropsychologique. 


\section{2) Coordonnées stéréotaxiques barycentriques des PPN}

Les coordonnées stéréotaxiques barycentriques moyennes recueillies en fonction de la ligne CA-CP pour l'ensemble des patients étaient les suivantes: le PPN rostral était situé en moyenne à $6,58 \mathrm{~mm}( \pm 0,73)$ en latéralité, $2,92 \mathrm{~mm}( \pm 0,78)$ derrière $\mathrm{CP}$ et $8,87 \mathrm{~mm}( \pm 0,55)$ sous la ligne CA-CP, le PPN caudal était situé en moyenne à 7,18 mm $( \pm 0,67)$ en latéralité, $4,07 \mathrm{~mm}( \pm 0,88)$ derrière $\mathrm{CP}$ et $13,18 \mathrm{~mm}( \pm 0,79)$ sous la ligne CA-CP.

Les moyennes des coordonnées de notre étude et de celles de Zrinzo et al. figurent dans le Tableau 2.

\begin{tabular}{|l|c|c|c|c|c|c|}
\hline & \multicolumn{3}{|c}{ PPN rostral } & \multicolumn{3}{c|}{ PPN caudal } \\
\hline Notre étude & $6,58( \pm 0,73)$ & $-2,92( \pm 0,78)$ & $-8,87( \pm 0,55)$ & $7,18( \pm 0,67)$ & $-4,07( \pm 0,88)$ & $-13,18( \pm 0,79)$ \\
\hline Zrinzo et al. & $6( \pm 0,5)$ & $-3( \pm 1,1)$ & $-9( \pm 1,1)$ & $6,8( \pm 0,5)$ & $-4( \pm 1,1)$ & $-13,9( \pm 1,2)$ \\
\hline Différence & 0,58 & 0,08 & 0,13 & 0,38 & 0,07 & 0,72 \\
\hline
\end{tabular}

Tableau 2: Coordonnées stéréotaxiques tridimensionnelles moyennes de l'ensemble des patients de notre étude et de celles de Zrinzo et al.

Moyennes ( \pm écart-types)

Les coordonnées sont en millimètres, en fonction de la ligne CA-CP.

$P P N$ : noyau pédonculo-pontin, LAT : latéralité, AP : antéro-postérieur, VERT : verticalité.

Les coordonnées stéréotaxiques barycentriques de l'ensemble de nos patients sont représentées dans le Tableau 3 avec test statistique comparativement aux coordonnées de Zrinzo et al. La schématisation tridimensionnelle des coordonnées est représentée dans la Figure 3. Les Figures 4 et 5 représentent, sous la forme de box plots les coordonnées barycentriques des PPN rostraux et caudaux de l'ensemble de nos patients ainsi que celles recueillies par Zrinzo et al.

Lorsqu'une différence a été mise en évidence par les tests statistiques, celle-ci est inférieure à $1 \mathrm{~mm}$. La différence maximale est observée pour la coordonnée de verticalité du PPN caudal, elle est de 0,72 millimètre. Les écart-types sont moins importants que ceux observés dans l'étude de Zrinzo et al., sauf pour les coordonnées de latéralité du PPN rostral et caudal (respectivement 0,73 versus 0,5 et 0,67 versus 0,5 ). 


\begin{tabular}{|c|c|c|c|c|c|c|c|}
\hline & \multicolumn{3}{|c|}{ PPN rostral } & \multicolumn{3}{|c|}{ PPN caudal } \\
\hline & & LAT & $\mathbf{A P}$ & VERT & LAT & $\mathbf{A P}$ & VERT \\
\hline 1 & DROITE & 5,73 & $-3,11$ & $-8,94$ & 6,76 & $-3,7$ & $-12,94$ \\
\hline 2 & DROITE & 6,11 & $-4,1$ & $-9,36$ & 6,34 & $-4,69$ & $-13,9$ \\
\hline 3 & DROITE & 6,53 & $-3,36$ & -9 & 7,36 & $-5,34$ & $-12,9$ \\
\hline 4 & DROITE & 6 & -3 & -9 & 7,46 & $-3,94$ & $-13,49$ \\
\hline 5 & DROITE & 5,3 & $-3,7$ & -9 & 7,15 & $-5,32$ & $-13,9$ \\
\hline 6 & DROITE & 5,82 & $-4,25$ & -9 & 5,73 & $-5,25$ & $-13,9$ \\
\hline 7 & DROITE & 6,83 & $-1,31$ & -8 & 7,44 & $-3,8$ & $-13,9$ \\
\hline 8 & DROITE & 4,89 & $-1,29$ & -8 & 5,82 & $-4,15$ & $-12,9$ \\
\hline 9 & DROITE & 6,15 & $-2,3$ & -10 & 6,52 & $-2,94$ & $-12,9$ \\
\hline 10 & DROITE & 5,92 & $-1,79$ & -9 & & & \\
\hline 11 & DROITE & 6,61 & $-3,21$ & -9 & 6,7 & $-5,03$ & $-13,9$ \\
\hline 12 & DROITE & 6,82 & -3 & -9 & 6,6 & $-3,59$ & $-12,9$ \\
\hline 13 & DROITE & 7,5 & -3 & -9 & 7,48 & $-2,61$ & $-12,9$ \\
\hline 14 & DROITE & 5,92 & $-2,13$ & $-8,07$ & 6,76 & $-3,13$ & $-13,9$ \\
\hline 15 & DROITE & 6,31 & $-2,79$ & -9 & 6,65 & $-3,78$ & $-11,9$ \\
\hline 16 & DROITE & 6,46 & $-3,18$ & -9 & 6,96 & $-5,38$ & $-11,9$ \\
\hline 17 & DROITE & 6,68 & $-2,24$ & -8 & 6,27 & $-3,7$ & $-12,9$ \\
\hline 18 & DROITE & 6,06 & $-3,25$ & -9 & 7,05 & $-4,37$ & $-13,9$ \\
\hline 19 & DROITE & 6,87 & $-3,25$ & -8 & 7,98 & -4 & $-14,9$ \\
\hline 20 & DROITE & 7,29 & $-3,16$ & $-8,41$ & 6,88 & $-4,64$ & $-13,31$ \\
\hline 21 & DROITE & 6,62 & -3 & -10 & 8,09 & $-4,89$ & $-13,9$ \\
\hline 22 & DROITE & 6,2 & $-3,91$ & -9 & & & \\
\hline 23 & DROITE & 6,02 & $-2,83$ & $-7,75$ & & & \\
\hline 24 & DROITE & 6 & $-3,69$ & -10 & 7,26 & $-3,07$ & $-13,9$ \\
\hline 25 & DROITE & 7,29 & $-2,22$ & -8 & 8,54 & $-3,54$ & $-12,9$ \\
\hline 26 & DROITE & 7,3 & $-1,24$ & -8 & 7,28 & $-3,34$ & $-12,9$ \\
\hline 27 & DROITE & 5,87 & $-2,61$ & $-8,9$ & 6,57 & $-3,54$ & $-13,9$ \\
\hline 28 & DROITE & 6,54 & -4 & -9 & 7,26 & $-4,52$ & $-11,9$ \\
\hline 29 & DROITE & 7 & -3 & -8 & 8,15 & $-3,74$ & $-12,9$ \\
\hline 30 & DROITE & 7,02 & $-3,2$ & -9 & 6,8 & $-2,98$ & $-12,9$ \\
\hline 1 & GAUCHE & 5,39 & $-3,06$ & $-9,04$ & 6,39 & $-2,52$ & $-12,06$ \\
\hline 2 & GAUCHE & 7,33 & $-4,71$ & $-9,36$ & 8,6 & $-3,48$ & $-11,95$ \\
\hline 3 & GAUCHE & 5,85 & $-3,3$ & -9 & 6,09 & $-3,82$ & $-13,9$ \\
\hline 4 & GAUCHE & 6,69 & -3 & -9 & 7,49 & $-3,46$ & $-13,9$ \\
\hline 5 & GAUCHE & 7,6 & $-4,8$ & -9 & 7,81 & $-6,84$ & $-13,9$ \\
\hline 6 & GAUCHE & & & & & & \\
\hline 7 & GAUCHE & 7,43 & $-2,39$ & -8 & 7,6 & $-4,54$ & $-13,9$ \\
\hline 8 & GAUCHE & 5,54 & $-1,49$ & -9 & 7,97 & $-2,79$ & $-11,9$ \\
\hline 9 & GAUCHE & 7,66 & $-2,85$ & -9 & 6,95 & $-2,94$ & $-12,9$ \\
\hline 10 & GAUCHE & 7,74 & $-2,24$ & -10 & & & \\
\hline 11 & GAUCHE & 7,33 & $-2,38$ & -9 & 7,7 & $-5,62$ & $-14,9$ \\
\hline 12 & GAUCHE & 6,69 & -3 & -9 & 6,65 & $-4,3$ & $-12,9$ \\
\hline 13 & GAUCHE & 6,35 & $-3,23$ & -9 & 6,92 & $-4,46$ & $-12,9$ \\
\hline 14 & GAUCHE & 5,37 & $-1,82$ & -9 & 6,35 & $-3,37$ & $-12,97$ \\
\hline 15 & GAUCHE & 7,64 & $-3,41$ & -9 & 8,75 & $-4,62$ & $-13,9$ \\
\hline 16 & GAUCHE & & & & & & \\
\hline 17 & GAUCHE & 6,76 & -3 & -9 & 7,63 & $-4,43$ & $-11,9$ \\
\hline 18 & GAUCHE & 6,98 & $-2,85$ & -9 & 6,92 & -5 & $-12,9$ \\
\hline 19 & GAUCHE & 5,56 & $-3,37$ & -8 & & & \\
\hline 20 & GAUCHE & 6,88 & $-3,64$ & -9 & 7,46 & $-4,26$ & $-13,9$ \\
\hline 21 & GAUCHE & 6,36 & $-2,64$ & -9 & 6,97 & $-5,01$ & $-12,9$ \\
\hline 22 & GAUCHE & 7,64 & $-3,37$ & -9 & & & \\
\hline 23 & GAUCHE & 8,18 & $-2,75$ & $-8,38$ & & & \\
\hline 24 & GAUCHE & 6,53 & $-3,71$ & -9 & 7,63 & $-3,63$ & $-13,9$ \\
\hline 25 & GAUCHE & 5,88 & $-2,7$ & -9 & 7,15 & -4 & $-12,9$ \\
\hline 26 & GAUCHE & 6,93 & $-1,38$ & -8 & 7,26 & $-4,23$ & $-12,9$ \\
\hline 27 & GAUCHE & 7,62 & $-2,31$ & -9 & 7,73 & $-2,84$ & $-11,9$ \\
\hline 28 & GAUCHE & 6,26 & $-3,26$ & -9 & 6,86 & $-4,26$ & $-12,9$ \\
\hline 29 & GAUCHE & 7,03 & $-2,74$ & $-9,9$ & 7,61 & $-3,84$ & $-11,9$ \\
\hline 30 & GAUCHE & 7,02 & -3 & -9 & 7,62 & $-4,2$ & $-13,9$ \\
\hline \multicolumn{2}{|c|}{ MOYENNE } & 6,58 & $-2,92$ & $-8,87$ & 7,18 & $-4,07$ & $-13,18$ \\
\hline \multicolumn{2}{|c|}{ ECART TYPE } & 0,73 & $\mathbf{0 , 7 8}$ & 0,55 & 0,67 & $\mathbf{0 , 8 8}$ & 0,79 \\
\hline \multicolumn{2}{|l|}{ p } & 0,0006 & 0,61 & 0,62 & 0,006 & 0,87 & 0,01 \\
\hline
\end{tabular}


Tableau 3 : Coordonnées stéréotaxiques barycentriques tridimensionnelles de l'ensemble des patients avec comparaison aux coordonnées de Zrinzo et al.

Les cases grisées, sans coordonnées, correspondent aux PPN dont les coordonnées étaient impossibles à relever. La numérotation des patients a été réalisée par ordre alphabétique. Les données sont mesurées en millimètres, en fonction de la ligne CA-CP.

$P P N$ : noyau pédonculo-pontin, LAT : latéralité, AP : antéro-postérieur, VERT : verticalité.

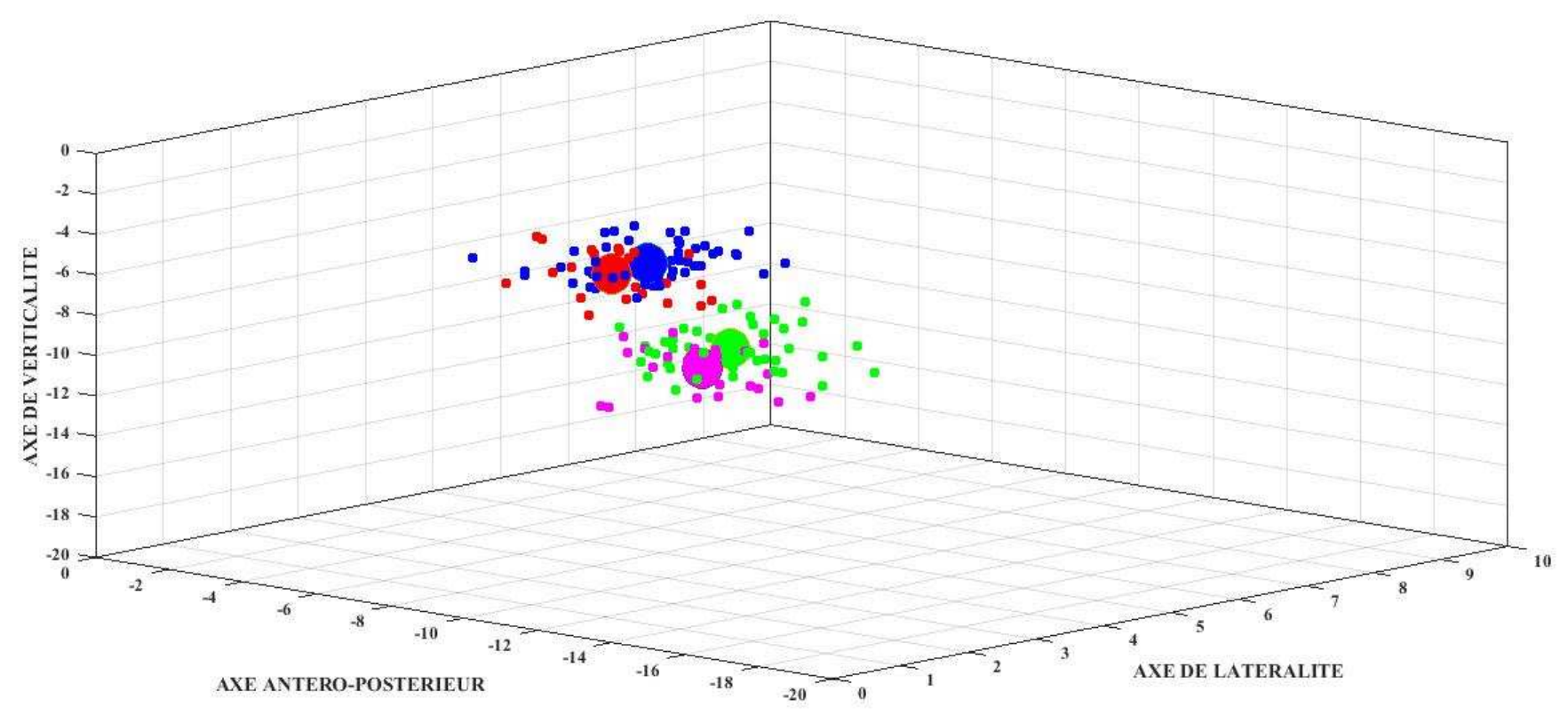

Figure 3: Schéma tridimensionnel représentant les coordonnées stéréotaxiques des PPN de l'ensemble des patients comparativement à celles de Zrinzo et al.

Les points bleus et verts correspondent respectivement aux coordonnées barycentriques des PPN rostraux et caudaux des patients issus de notre étude. Les points rouges et roses correspondent respectivement aux coordonnées barycentriques des PPN rostraux et caudaux de l'étude de Zrinzo et al. Les points plus volumineux correspondent aux moyennes des coordonnées. Les 3 axes sont mesurés en millimètres en fonction de la ligne $C A-C P$. 


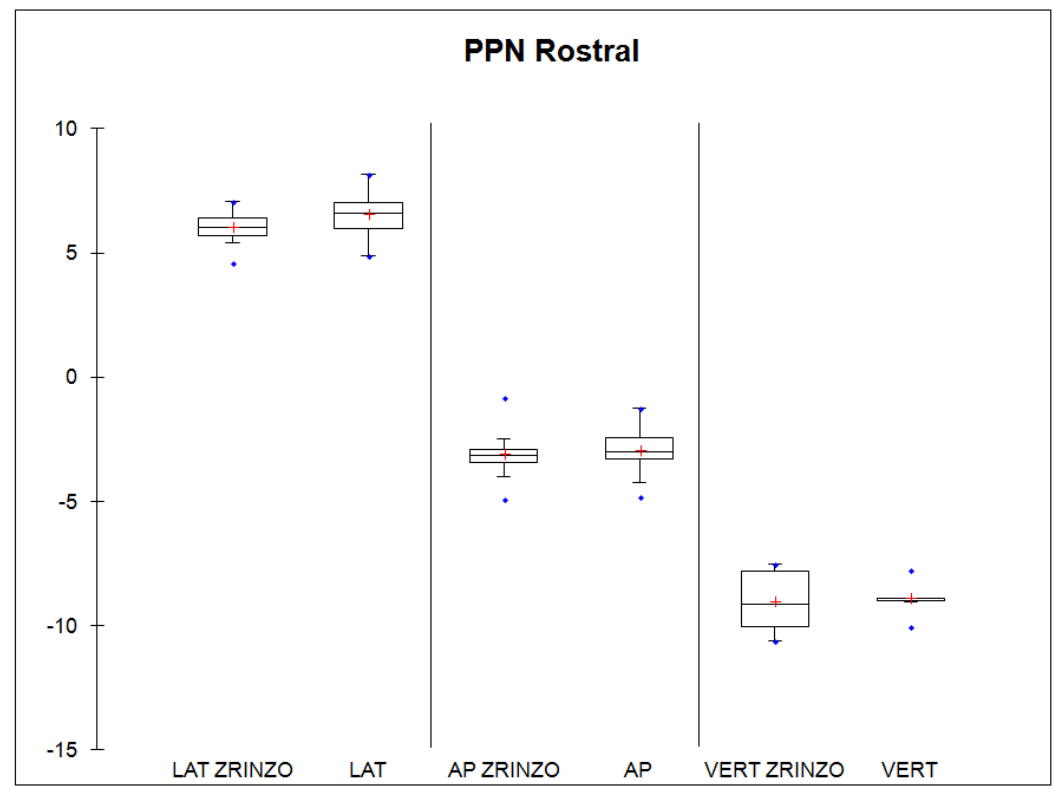

Figure 4: Box plots représentant les coordonnées barycentriques des PPN rostraux de l'ensemble de nos patients ainsi que celles de Zrinzo et al.

$P P N$ : noyau pédonculo-pontin, LAT : coordonnée de latéralité, AP : coordonnée antéro-postérieure, VERT : coordonnée de verticalité.

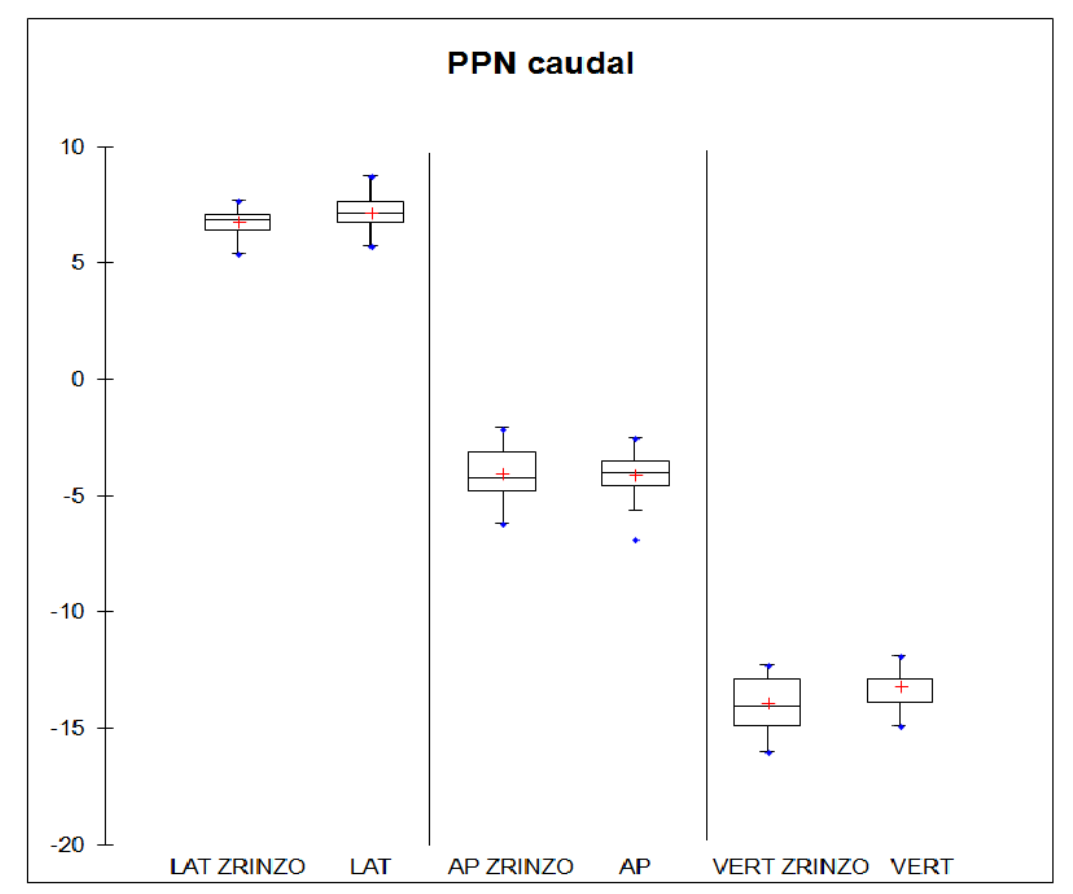

Figure 5: Box plots représentant les coordonnées barycentriques des PPN caudaux de l'ensemble de nos patients ainsi que celles de Zrinzo et al.

$P P N$ : noyau pédonculo-pontin, LAT : coordonnée de latéralité, AP : coordonnée antéro-postérieure, VERT : coordonnée de verticalité. 
Les coordonnées des PPN du groupe contrôle figurent dans le Tableau 4 avec test statistique comparativement aux coordonnées de Zrinzo et al.

\begin{tabular}{|l|l|r|r|r|r|r|r|}
\hline & \multicolumn{3}{|c|}{ PPN rostral } & \multicolumn{3}{c|}{ PPN caudal } \\
\hline Contrôle 1 & DROIT & \multicolumn{1}{c|}{ LAT } & \multicolumn{1}{c|}{ AP } & \multicolumn{1}{c|}{ VERT } & \multicolumn{1}{c|}{ LAT } & \multicolumn{1}{c|}{ AP } & \multicolumn{1}{c|}{ VERT } \\
\hline Contrôle 2 & DROIT & 5,73 & $-3,11$ & $-8,94$ & 6,76 & $-3,7$ & $-12,94$ \\
\hline Contrôle 3 & DROIT & 6,11 & $-4,1$ & $-9,36$ & 6,34 & $-4,69$ & $-13,9$ \\
\hline Contrôle 4 & DROIT & 6 & $-3,36$ & -9 & 7,36 & $-5,34$ & $-12,9$ \\
\hline Contrôle 5 & DROIT & 5,3 & $-3,7$ & -9 & 7,15 & $-5,32$ & $-13,49$ \\
\hline Contrôle 1 & GAUCHE & 5,39 & $-3,06$ & $-9,04$ & 6,39 & $-2,52$ & $-12,06$ \\
\hline Contrôle 2 & GAUCHE & 7,33 & $-4,71$ & $-9,36$ & 8,6 & $-3,48$ & $-11,95$ \\
\hline Contrôle 3 & GAUCHE & 5,85 & $-3,3$ & -9 & 6,09 & $-3,82$ & $-13,9$ \\
\hline Contrôle 4 & GAUCHE & 6,69 & -3 & -9 & 7,49 & $-3,46$ & $-13,9$ \\
\hline Contrôle 5 & GAUCHE & 7,6 & $-4,8$ & -9 & 7,81 & $-6,84$ & $-13,9$ \\
\hline MOYENNE & & $\mathbf{6 , 2 5}$ & $\mathbf{- 3 , 6 1}$ & $\mathbf{- 9 , 0 7}$ & $\mathbf{7 , 1 5}$ & $\mathbf{- 4 , 3 1}$ & $\mathbf{- 1 3 , 2 8}$ \\
\hline ECART TYPE & & $\mathbf{0 , 7 8}$ & $\mathbf{0 , 6 9}$ & $\mathbf{0 , 1 5}$ & $\mathbf{0 , 7 7}$ & $\mathbf{1 , 2 5}$ & $\mathbf{0 , 7 8}$ \\
\hline p & & $\mathbf{0 , 4 7 3}$ & $\mathbf{0 , 0 7 7}$ & $\mathbf{0 , 7 1 2}$ & $\mathbf{0 , 1 7 9}$ & $\mathbf{0 , 5 3 9}$ & $\mathbf{0 , 0 8 2}$ \\
\hline
\end{tabular}

Tableau 4 : Coordonnées stéréotaxiques barycentriques tridimensionnelles des patients du groupe contrôle avec comparaison aux coordonnées de Zrinzo et al.

Les données sont mesurées en millimètres, en fonction de la ligne CA-CP. $P P N$ : noyau pédonculo-pontin, LAT : latéralité, AP : antéro-postérieur, VERT : verticalité.

\section{3) Corrélation}

Le test de Spearman a mis en évidence une forte corrélation entre les parties rostrales et caudales du PPN, ainsi qu'entre le PPN droit et le PPN gauche. Devant cette inter-dépendance des variables, nous n'avons donc pas pu pooler les différentes données et avons étudié de manière individuelle chaque partie du PPN : PPN rostral droit, PPN caudal droit, PPN rostral gauche et PPN caudal gauche. 


\section{4) Analyse semi-quantitative}

Pour chaque patient, l'évaluation de la taille du PNN a pu être réalisée pour les parties rostrale et caudale selon les 4 catégories précédemment définies.

Parmi les 5 sujets contrôle, seuls 15\% d'entre eux ont un PPN évalué à 1 (atrophie légère). Toutes les autres évaluations sont à 0 (absence d'atrophie).

Dans le groupe FOG +, il existe davantage de PPN évalués à 2 ou à 3 comparativement au groupe FOG - (27\% versus $17 \%)$.

Toutefois, lorsque l'on compare les différents groupes entre eux, il n'a pas été mis en évidence de différence statistiquement significative concernant le degré d'atrophie du PPN pour les parties rostrales ou caudales, à droite comme à gauche $: \mathrm{p}=0,151$ pour le PPN rostral droit, $\mathrm{p}=0,169$ pour le PPN caudal droit, $\mathrm{p}=0,507$ pour le PPN rostral gauche et $\mathrm{p}=0,190$ pour le PPN caudal gauche.

Les différentes évaluations de la taille du PPN sont représentées dans le Tableau 5.

Les figures 6 et 7 illustrent les parties rostrale et caudale d'un PPN de taille normale d'un sujet du groupe contrôle.

Les figures 8 et 9 illustrent les parties rostrale et caudale d'un PPN présentant une atrophie modérée d'un patient du groupe FOG +. 


\begin{tabular}{|c|c|c|c|c|c|c|}
\hline & \multirow{2}{*}{$\begin{array}{c}\text { Age } \\
\text { (années) }\end{array}$} & \multirow{2}{*}{$\begin{array}{l}\text { Latéralité } \\
\text { syndrome } \\
\text { parkinsonien }\end{array}$} & \multicolumn{2}{|c|}{ DROITE } & \multicolumn{2}{|c|}{ GAUCHE } \\
\hline & & & $\begin{array}{c}\text { PPN } \\
\text { rostral }\end{array}$ & $\begin{array}{c}\text { PPN } \\
\text { caudal }\end{array}$ & $\begin{array}{c}\text { PPN } \\
\text { rostral }\end{array}$ & $\begin{array}{c}\text { PPN } \\
\text { caudal }\end{array}$ \\
\hline FOG + 1 & 63 & Gauche & 1 & 2 & 2 & 1 \\
\hline FOG +2 & 63 & Gauche & 2 & 2 & 0 & 1 \\
\hline FOG +3 & 67 & Droite & 1 & 0 & 0 & 1 \\
\hline FOG +4 & 76 & Gauche & 2 & 1 & 2 & 1 \\
\hline FOG +5 & 52 & Gauche & 1 & 3 & 1 & 3 \\
\hline FOG + 6 & 66 & Droite & 1 & 0 & 1 & 0 \\
\hline FOG + 7 & 67 & Gauche & 0 & 1 & 1 & 0 \\
\hline FOG +8 & 66 & Gauche & 0 & 0 & 0 & 0 \\
\hline FOG +9 & 65 & Gauche & 0 & 0 & 0 & 0 \\
\hline FOG + 10 & 71 & Gauche & 2 & 2 & 1 & 0 \\
\hline FOG + 11 & 74 & Droite & 2 & 0 & 2 & 3 \\
\hline FOG + 12 & 54 & Gauche & 0 & 0 & 0 & 0 \\
\hline FOG - 1 & 60 & Droite & 0 & 0 & 0 & 0 \\
\hline FOG - 2 & 62 & Gauche & 1 & 0 & 0 & 3 \\
\hline FOG - 3 & 47 & Gauche & 0 & 0 & 0 & 0 \\
\hline FOG - 4 & 68 & Droite & 0 & 0 & 0 & 0 \\
\hline FOG - 5 & 68 & Droite & 2 & 3 & 2 & 3 \\
\hline FOG - 6 & 78 & Droite & 2 & 3 & 2 & 3 \\
\hline FOG - 7 & 55 & Droite & 0 & 0 & 0 & 0 \\
\hline FOG - 8 & 57 & Droite & 1 & 1 & 0 & 1 \\
\hline FOG - 9 & 61 & Droite & 1 & 1 & 1 & 1 \\
\hline FOG - 10 & 56 & Droite & 0 & 1 & 1 & 0 \\
\hline FOG - 11 & 68 & Gauche & 0 & 0 & 0 & 0 \\
\hline FOG - 12 & 53 & Droite & 1 & 0 & 1 & 0 \\
\hline FOG - 13 & 55 & Gauche & 0 & 0 & 0 & 0 \\
\hline CONTRÔLE 1 & 63 & NA & 0 & 0 & 0 & 0 \\
\hline CONTRÔLE 2 & 66 & NA & 1 & 0 & 0 & 0 \\
\hline CONTRÔLE 3 & 44 & NA & 0 & 0 & 1 & 0 \\
\hline CONTRÔLE 4 & 29 & NA & 0 & 0 & 0 & 0 \\
\hline CONTRÔLE 5 & 54 & NA & 0 & 0 & 1 & 0 \\
\hline
\end{tabular}

Tableau 5 : Evaluations semi-quantitatives de l'ensemble des patients de notre étude.

$0=$ pas d'atrophie, $1=$ atrophie légère, $2=$ atrophie modérée, $3=$ atrophie sévère ou PPN non visible, PPN : noyau pédonculo-pontin, FOG + : freezer, FOG - : non freezer, NA : non applicable. 


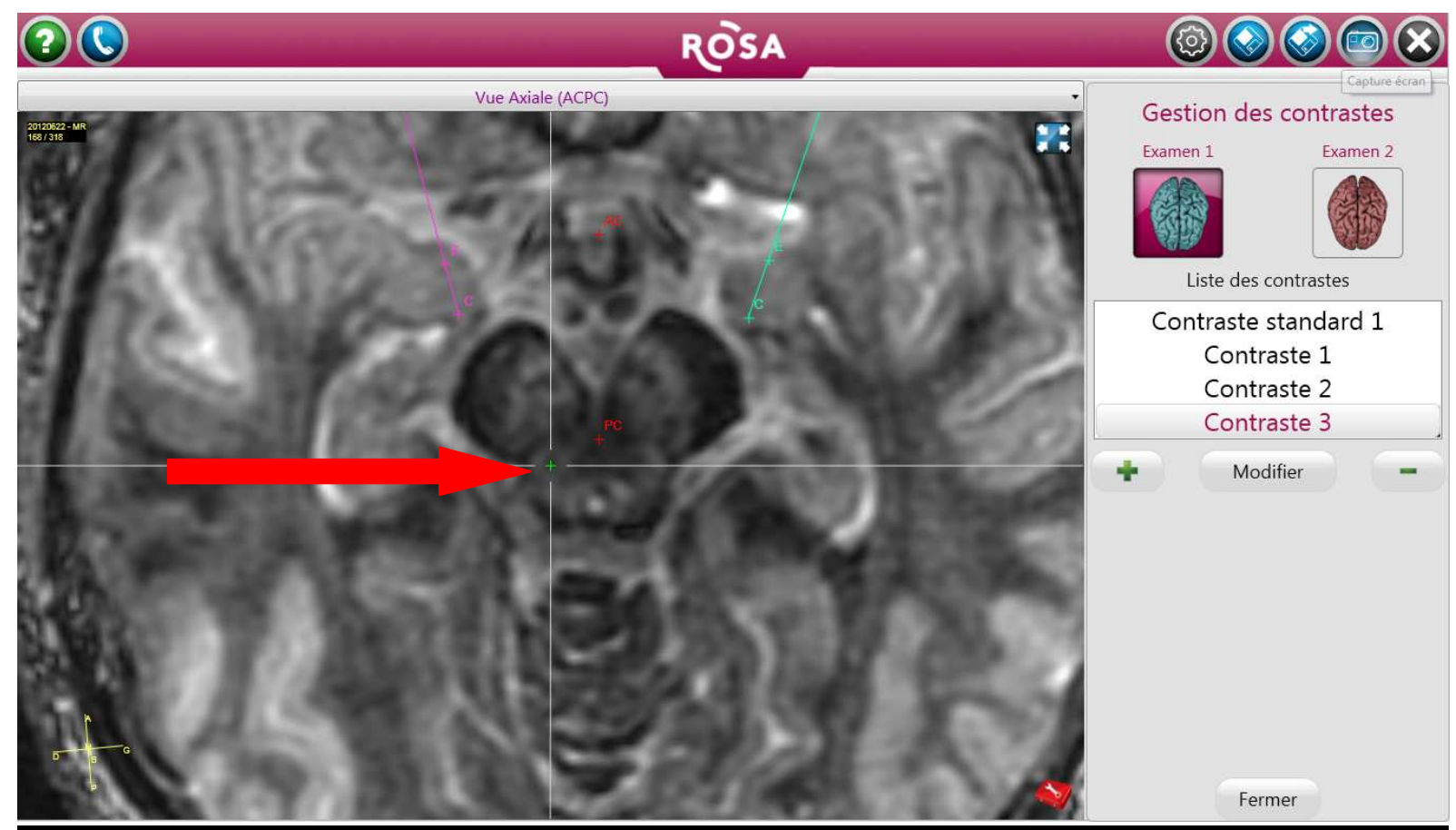

Figure 6: séquence FGATIR modifiée d'une IRM cérébrale en coupe axiale, image issue du logiciel ROSANNA visualisant un PPN rostral droit de taille normale, évaluation semiquantitative : 0 (absence d'atrophie), chez un patient du groupe contrôle.

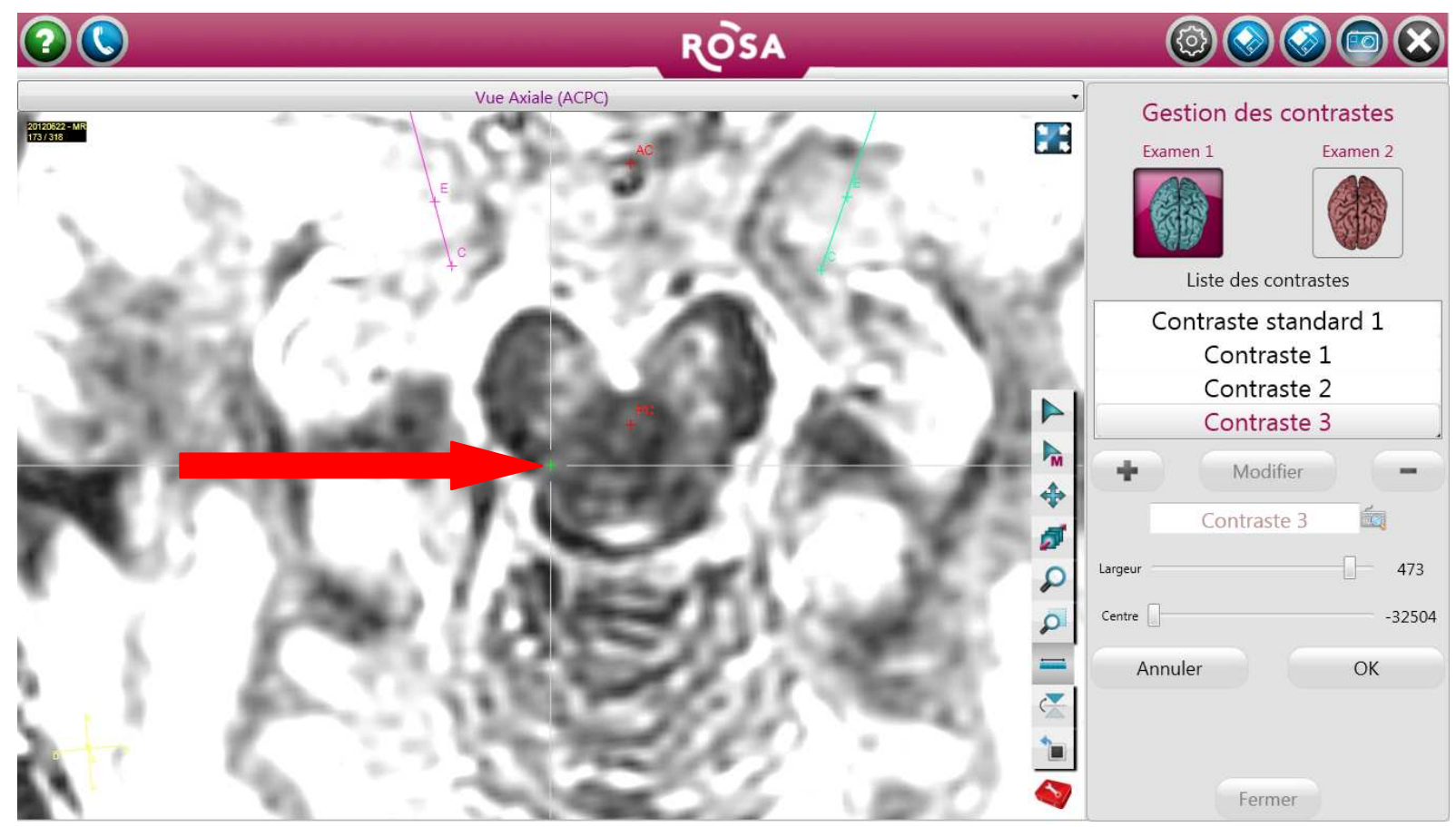

Figure 7 : séquence FGATIR modifiée d'une IRM cérébrale en coupe axiale, image issue du logiciel ROSANNA visualisant un PPN caudal droit de taille normale, évaluation semiquantitative : 0 (absence d'atrophie), chez le même patient du groupe contrôle qu'en figure 6 . 


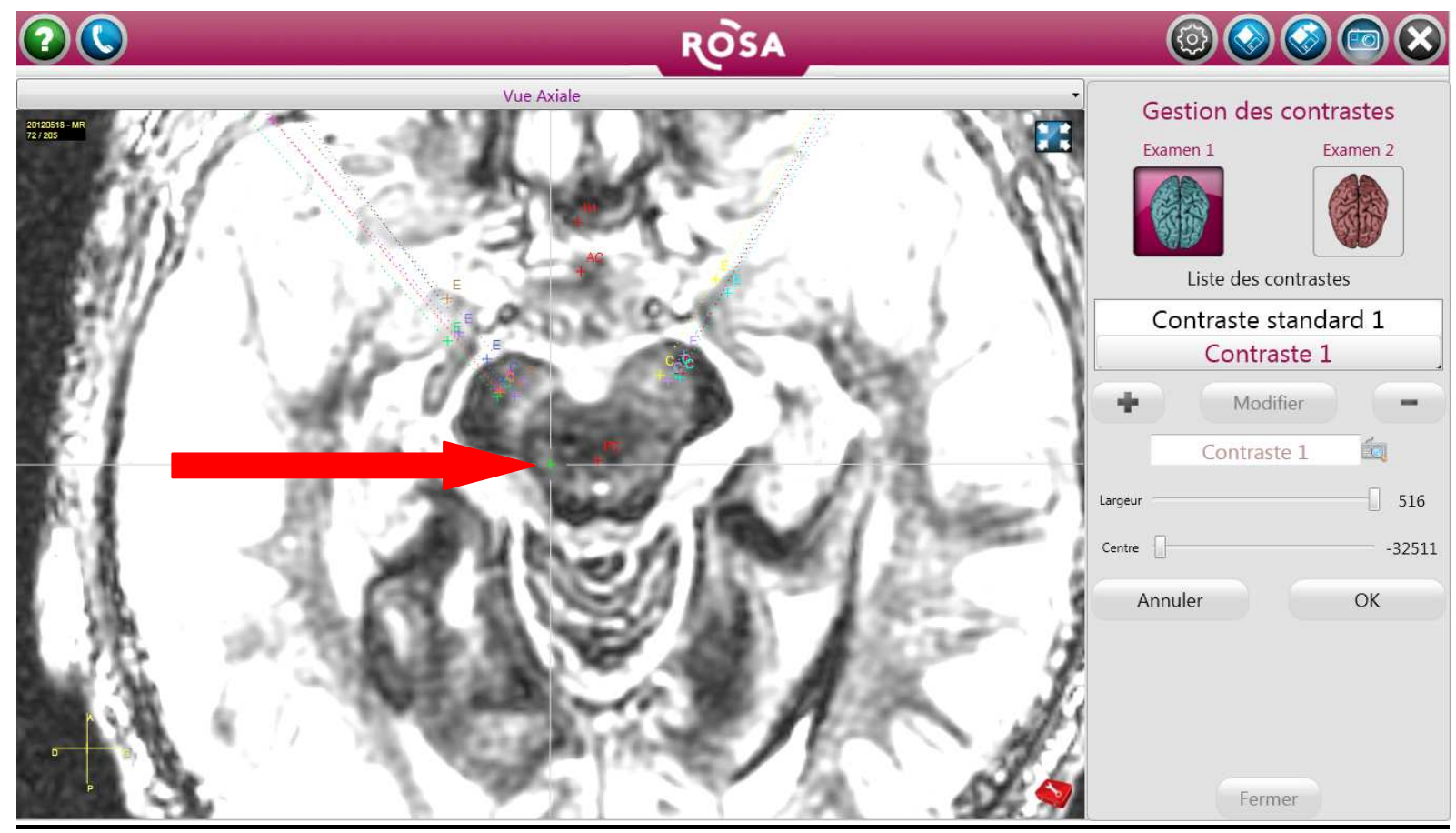

Figure 8 : séquence FGATIR modifiée d'une IRM cérébrale en coupe axiale, image issue du logiciel ROSANNA visualisant un PPN rostral droit de taille réduite, évaluation semiquantitative : 2 (atrophie modérée), chez un patient du groupe FOG + .

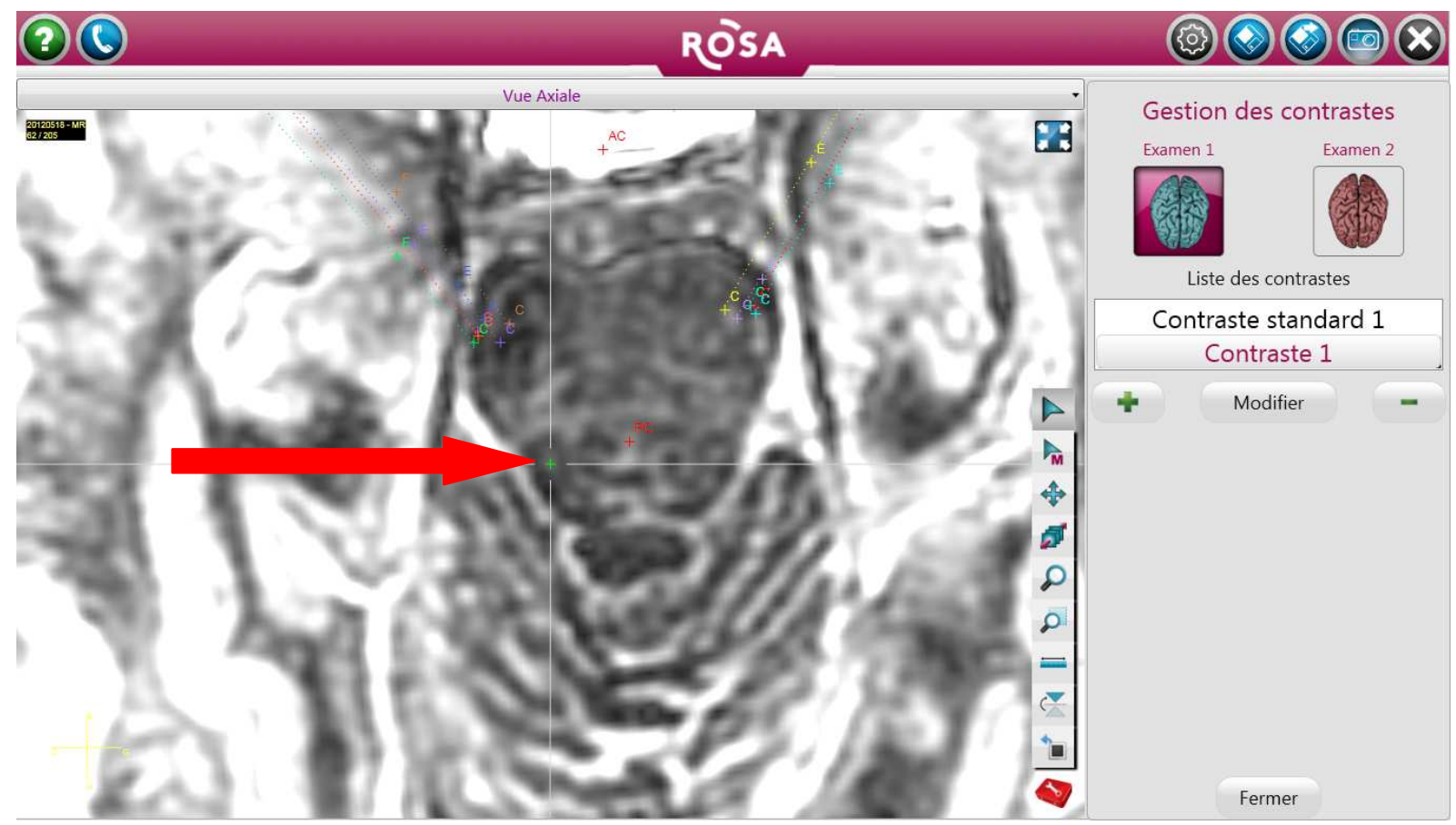

Figure 9: séquence FGATIR modifiée, image issue du logiciel ROSANNA visualisant un PPN caudal droit de taille réduite, évaluation semi-quantitative : 2 (atrophie modérée), chez le même patient du groupe FOG + qu'en figure 8 . 


\section{5) Fiabilité intra-examinateur}

Il n'existe pas de différence significative entre la première et la deuxième évaluation semi quantitative concernant la partie rostrale des PPN droits et gauches (respectivement Kappa = $1, \mathrm{p}<0,0001$ et Kappa $=0,756, \mathrm{p}=0,001)$ ainsi que pour la partie caudale des PPN droits et gauches (respectivement Kappa =0,63, p =0,005 et Kappa =0,29, p =0,035).

\section{6) Fiabilité inter-examinateur}

Il n'existe pas de différence significative entre l'évaluation semi-quantitative de la taille du PPN réalisée par l'examinateur numéro 1 (neurologue) et celle réalisée par l'examinateur numéro 2 (neurochirurgien) concernant la partie rostrale des PPN droits et gauches (respectivement Kappa $=1, \mathrm{p}<0,0001$ et Kappa $=0,756, \mathrm{p}=0,001)$ et la partie caudale des PPN droits (Kappa $=1, \mathrm{p}<0,0001)$.

Toutefois, nous n'avons pas pu mettre en évidence de corrélation inter-examinateur concernant la partie caudale des PPN gauches (Kappa $=0,268, \mathrm{p}=0,247$ ).

\section{7) Critères secondaires}

Il n'existe pas de corrélation significative entre d'une part la taille du PPN et d'autre part le sous score axial composite de l'UPDRS III (conditions worst Off et best On), les données du bilan neuropsychologique concernant la MADRS et la LARS. On note une corrélation entre le score à la DRS de Mattis et la taille du PPN caudal gauche $(p=0,011)$. Les résultats figurent dans le Tableau 6.

\begin{tabular}{|l|ccccc|}
\hline & $\begin{array}{c}\text { Sous score axial } \\
\text { worst Off }\end{array}$ & $\begin{array}{c}\text { Sous score axial } \\
\text { best On }\end{array}$ & DRS de Mattis & MADRS & LARS \\
\hline PPNr dt & 0,243 & 0,334 & 0,573 & 0,939 & 0,06 \\
\hline PPNr g & 0,256 & 0,024 & 0,586 & 0,992 & 0,013 \\
\hline PPNc dt & 0,09 & 0,171 & 0,158 & 0,13 & 0,315 \\
\hline PPNc g & 0,125 & 0,08 & 0,011 & 0,699 & 0,702 \\
\hline
\end{tabular}

Tableau 6 : Corrélation entre la taille du PPN et les critères secondaires.

PPNr : noyau pédonculo-pontin rostral, PPNc: noyau pédonculo-pontin caudal, dt: droit, $g$ : gauche, DRS : Dementia Rating Scale, MADRS : Montgomery and Asberg Depression Rating Scale, LARS : Lille Apathy Rating Scale 


\section{V) Discussion}

Le but de cette étude était de rechercher une corrélation entre l'atrophie du PPN et la présence ou non d'un freezing chez les patients présentant une maladie de Parkinson. Pour y arriver, plusieurs étapes ont été nécessaires.

La première étape consistait à recueillir les coordonnées barycentriques des PPN de l'ensemble des patients inclus dans notre étude et de les comparer à celles définies par Zrinzo et al. [22]. Cette étape était cruciale dans notre démarche car nous devions nous assurer que la structure étudiée correspondait véritablement au PPN. La deuxième étape reposait sur l'évaluation semi-quantitative du PPN. Cette étape était scindée en plusieurs parties. Nous devions tout d'abord nous assurer de la reproductibilité de l'échelle d'évaluation que nous avions choisi d'appliquer à la mesure du PPN, que ce soit entre deux examinateurs (fiabilité inter-examinateur) ou pour le même examinateur à quelques jours d'intervalle (fiabilité intraexaminateur). Et enfin, après ces vérifications, nous pouvions évaluer s'il existait ou non une corrélation entre le degré d'atrophie du PPN et la présence ou non d'un freezing.

Les coordonnées des PPN recueillies dans notre étude diffèrent pour certaines de manière significative des coordonnées recueillies par Zrinzo et al. Toutefois, lorsqu'il existe une différence significative mise en évidence par les tests statistiques, celle-ci diffère de moins d'un millimètre, la différence maximale observée est de 0,72 millimètre pour la coordonnée de verticalité du PPN caudal. Cela peut être en lien avec la différence de résolution lors de l'acquisition des séquences IRM. En effet, dans l'étude de Zrinzo et al. [22], le champ de l'IRM était de 1,5 Tesla et la résolution de 256×256 avec une épaisseur de coupes de $2 \mathrm{~mm}$. Dans notre étude, l'IRM utilisée était une 3 Teslas et la résolution était bien meilleure, puisque celle-ci était de $512 \times 512$ avec une épaisseur de coupes de $1 \mathrm{~mm}$. La différence suggérée par les résultats des tests statistiques est probablement liée à la différence d'épaisseur de coupes, à la qualité de la résolution spatiale et à une différence lors de l'établissement de la ligne CA-CP entre notre étude et celle de Zrinzo et al. Concernant les écart-types des moyennes, ceux-ci sont moins importants pour l'ensemble de nos coordonnées, hormis pour les coordonnées de latéralité des PPN rostraux et caudaux (respectivement 0,73 versus 0,5 et 0,67 versus 0,5 ), cela suggère une discrète variabilité inter individuelle des coordonnées des PPN. La structure que nous avons évaluée correspond donc véritablement au PPN dont les coordonnées ont été recueillies grâce à une excellente résolution lors de l'acquisition de la séquence FGATIR modifiée. 
Nous avons choisi de réaliser une évaluation semi-quantitative car il s'agit d'un outil simple de mesure. Rappelons que le PPN est considéré comme un amas cellulaire, les contours restent mal définis. Il nous était difficile de réaliser une quantification précise puisque les limites de cette structure étaient mal définies. Toutefois, grâce à une bonne résolution de la séquence IRM, il nous était possible de visualiser le PPN et de voir ses contours en miroir par rapport aux structures adjacentes. L'IRM est un outil accessible aux cliniciens en routine quotidienne, notamment au neurochirurgien dans un but de visée et au neurologue pour qui cet examen constitue un outil au lit du patient permettant une interprétation simple. Cependant, il faut souligner le fait que la visualisation de cette structure nécessite un apprentissage et une connaissance parfaite des structures qui l'entourent.

Il semblerait que dans notre population de patients, l'atrophie soit davantage marquée chez les patients âgés et/ou freezers. En effet, dans le groupe FOG +, davantage de PPN sont évalués à 2 ou à 3 comparativement au groupe FOG - (27\% versus 17\%). Il existe également une tendance à l'atrophie du PPN chez les patients âgés. L'atrophie corticale est vraisemblablement associée à une atrophie des structures sous corticales, comme le PPN. Lefranc et al. [33] ont rapporté le cas d'une patiente opérée au CHU d'Amiens d'une SCP des noyaux rouges dans le cadre d'un tremblement cérébelleux. Cette patiente avait à la fois une atrophie sévère des PPN, à peine visualisable et une atrophie corticale sévère d'origine alcoolo-carentielle.

Notre étude n'a pas pu mettre en évidence de différence statistiquement significative de la taille du PPN entre les différents groupes (FOG +, FOG - et le groupe contrôle). Cette absence de différence significative est probablement en lien avec le faible effectif dans chacun des groupes. L'autre explication reposerait sur le mode de recrutement de notre population. En effet, les patients sélectionnés et inclus dans notre étude (groupes FOG + et FOG -) étaient des patients qui allaient bénéficier d'une SCP des NST dont on sait que les critères d'exclusion sont la présence de signes axiaux marqués non dopa-sensibles du fait d'un risque d'aggravation en postopératoire. La majorité des patients du groupe FOG + étaient très peu freezers et répondaient pour la plupart, très bien au traitement dopaminergique. Le faible effectif dans notre étude s'explique par le fait que seuls les patients qui allaient être opérés d'une SCP pouvaient bénéficier de la séquence FGATIR modifiée dans le cadre du bilan préchirurgical. Il faut toutefois souligner que notre étude est une étude pilote qui met en avant la séquence IRM FGATIR modifiée, qui possède une bonne résolution et qui a permis de recueillir les coordonnées des PPN de manière très précise. Cette séquence permet également 
pour la première fois de réaliser une mesure de la taille du PPN grâce à sa bonne résolution. En améliorant davantage la résolution de la séquence, nous pourrions à terme réaliser une quantification précise de la taille du PPN.

Lors de l'évaluation de la taille du PPN, nous avons parfois remarqué une asymétrie de taille entre les PPN droit et gauche. Cette constatation concerne à plus forte raison le groupe FOG + dans lequel $50 \%$ des patients présentaient une asymétrie de taille. Chez ces patients, l'atrophie du PPN était plus importante du côté controlatéral au syndrome parkinsonien et concernait essentiellement la partie caudale.

Les fiabilités intra et inter-examinateurs sont globalement moins bonnes concernant la partie caudale du PPN. La partie caudale est en effet beaucoup moins volumineuse que la partie rostrale et donc bien plus difficile à visualiser. Il s'agit d'une structure extrêmement fine qui, du fait de sa position caudale, c'est-à-dire plus éloignée de la ligne CA-CP que la partie rostrale, est davantage susceptible aux effets de reformatage de l'image, avec une possible perte en résolution de contraste. L'évaluation semi-quantitative en quatre catégories que nous avons réalisée était purement visuelle et n'est probablement pas adaptée à cette partie du PPN. Il était en effet difficile de réaliser une évaluation en quatre catégories sur une structure ne mesurant que quelques millimètres. Toutefois, lorsqu'il existait une différence d'évaluation inter ou intra-examinateur, celle-ci ne différait que d'une seule catégorie ( 0 à la place de 1 et inversement ou 2 à la place de 3 et inversement). Une évaluation en deux catégories aurait été probablement plus adaptée à la taille de cette partie du noyau. C'est la raison pour laquelle les reproductibilités intra et inter examinateurs sont moins bonnes. Toutefois, malgré des résultats statistiques ne retrouvant pas de bonne reproductibilité inter-examinateur pour le PPN caudal gauche (Kappa =0,268, $\mathrm{p}=0$ 0,247), la reproductibilité a été parfaite pour 7 des 10 patients. Par ailleurs, si la classification en deux catégories avait été utilisée, les reproductibilités inter et intra-examinateurs auraient été parfaites avec une reproductibilité de $100 \%$ de manière systématique pour les PPN caudaux. A contrario, l'évaluation choisie était tout à fait adaptée à la partie rostrale du PPN qui est parfaitement visible sur la séquence FGATIR modifiée et avec de bonnes reproductibilités inter et intra-examinateurs. De plus, notre séquence FGATIR modifiée pourrait être davantage optimisée dans la visualisation du PPN. Par exemple, en centrant la séquence sur le PPN, la perte de définition liée au reformatage de l'image serait moindre. La résolution serait alors améliorée, permettant une meilleure visualisation du PPN, notamment la partie caudale. Rappelons que la séquence que nous avons utilisée n'était pas 
spécifiquement dédiée à la visualisation du PPN mais qu'elle rentrait dans le cadre de l'IRM préopératoire d'une SCP dont la cible n'était jamais le PPN.

Nous avons également observé une moins bonne reproductibilité globale des évaluations sur les parties gauches du PPN. Les évaluations ont débuté systématiquement à droite pour l'ensemble des cotations. Il faudrait à l'avenir refaire ces mêmes évaluations en débutant par la gauche et vérifier si les résultats sont superposables.

Concernant les critères secondaires, il semble exister une corrélation entre le degré d'atrophie du PPN caudal gauche et les scores obtenus à la DRS de Mattis $(\mathrm{p}=0,011)$. Les patients dont le PPN caudal gauche était côté à 3 avaient une DRS de Mattis moyenne à 133,6 ( $\pm 3,21)$. On observait de plus un score plus élevé pour les patients sans atrophie du PPN ou ayant une atrophie légère, respectivement $137,5( \pm 3,80)$ et $140,5( \pm 1,97)$. Ces résultats semblent surprenants probablement du fait d'un petit effectif de nos populations. Ils ne concernent que le PPN caudal gauche. Rappelons que la partie caudale est bien plus difficile à visualiser du fait de sa très petite taille et de sa position (possible perte en résolution de contraste), cela a pour conséquence une évaluation semi-quantitative beaucoup moins aisée. Afin de confirmer ces premiers résultats, il faudra donc par la suite augmenter l'effectif en incluant davantage de patients.

Si les tendances évoquées par les résultats de notre étude se confirment avec une population de plus grande envergure et ne présentant pas de biais de sélection, une taille réduite du PPN, pourrait être évaluée comme facteur prédictif d'apparition de signes axiaux en post-opératoire d'une SCP des NST comme le suggérait le travail de Rouaud et al. [34]. En effet, le seul facteur prédictif de bon résultat après une SCP des NST, reconnu actuellement, repose sur une bonne dopa-sensibilité préopératoire [35]. Il s'agit d'une préoccupation de premier ordre dans la maladie de Parkinson comme en témoigne notamment le protocole hospitalier de recherche national PREDI-STIM (Dr D. Devos, CHRU Lille) actuellement en cours qui a pour objectif de mettre en évidence l'ensemble des facteurs prédictifs de réponse de la SCP des NST.

Le PPN reste donc une structure de grand intérêt puisque sa physiopathologie n'est que partiellement élucidée. Elle constitue également pour certains une cible de SCP dont il faut définir de manière très précise les critères d'éligibilité et choisir des critères objectifs d'évaluation. De plus, avec la séquence que nous avons utilisée, nous obtenons une meilleure visualisation du PPN, le ciblage de cette structure sera donc plus aisé avec cette séquence, qui reste tout de même à optimiser, notamment pour la partie caudale de ce noyau. Si l'ensemble 
de ces critères sont réunis, nous pourrions espérer des résultats plus homogènes à la SCP du PPN.

\section{VI) Conclusion}

Notre étude permet de bien montrer l'intérêt de la séquence FGATIR modifiée pour la localisation précise du PPN. Grâce à sa bonne résolution, nous avons pu réaliser des mesures semi-quantitatives reproductibles de la taille du PPN. Des différences de tailles ont pu être observées dans notre population avec une tendance à l'atrophie chez les patients freezers. Le but sera donc de former un utilisateur lambda en peu de temps (15 minutes par exemple) afin que cette méthode soit utilisée en routine. Avec l'optimisation de la séquence et une population de plus grande envergure, il serait intéressant d'évaluer l'atrophie du PPN comme facteur prédictif d'aggravation des signes axiaux en post-opératoire d'une SCP des NST chez les patients parkinsoniens. 


\section{Références bibliographiques}

1. De Rijk MC, Launer LJ, Berger K, Breteler MM, Dartigues JF, Baldereschi M, et al. Prevalence of Parkinson's disease in Europe : A collaborative study of populationbased cohorts. Neurologic Diseases in the Elderly Research Group Neurology 2000 ; 54 (11supp15):S21-S23

2. Macht M, Kaussner Y, Möller JC, Stiasny-Kolster K, Eggert KM, Krüger HP, et al. Predictors of freezing in Parkinson's disease : a survey of 6,620 patients. Mov. Disord. 2007; 22(7):953-6

3. Bloem BR, Hausdorff JM, Visser JE, Giladi N. Falls and freezing of gait in Parkinson's disease : a review of two interconnected, episodic phenomena. Mov Disord 2004; 19:871-84.

4. Schaafsma JD, Giladi N, Balash Y, Bartels AL, Gurevich T, Hausdorff JM. Gait dynamics in Parkinson's disease : relationship to Parkinsonian features, falls and response to levodopa. J Neurol Sci 2003; 212:47-53.

5. Amboni M, Cozzolino A, Longo K, Picillo M, Barone P. Freezing of gait and executive functions in patients with Parkinson's disease. Mov Disord 2008; 23:395400.

6. Giladi N, McMahon D, Przedborski S, Flaster E, Guillory S, Kostic V, et al. Motor blocks in Parkinson's disease. Neurology 1992; 42: 333-9.

7. Giladi N, Treves TA, Simon ES, Shabtai H, Orlov Y, Kandinov B, et al. Freezing of gait in patients with advanced Parkinson's disease. J Neural Transm 2001a; 108:53-61

8. Hirsch EC, Graybiel AM, Duyckaerts C, Javoy-Agid F. Neuronal loss in the pedunculopontine tegmental nucleus in Parkinson disease and in progressive supranuclear palsy. Proc Natl Acad Sci USA 1987; 84:5976 -5980

9. Zweig RM, Whitehouse PJ, Casanova MF, Walker LC, Jankel WR, Price DL. Loss of pedunculopontine neurons in progressive supranuclear palsy. Ann Neurol 1987; 22:18-25

10. Jellinger K. The pedunculopontine nucleus in Parkinson's disease, progressive supranuclear palsy and Alzheimer's disease. J Neurol Neurosurg Psychiatry 1988; 51:540-543

11. Olszewski J, Baxter D. Cytoarchitecture of the human brainstem, 1st ed. Philadelphia: Lippencott; 1954 
12. Jenkinson N, Nandi D, Muthusamy K, et al. Anatomy, physiology, and pathophysiology of the pedunculopontine nucleus. Mov Disord 2009; 24:319-328

13. Karachi C, Grabli D, Bernard FA, Tandé D, Wattiez N, Belaid H, et al. Cholinergic mesencephalic neurons are involved in gait and postural disorders in Parkinson disease. J Clin Invest 2010; 120:2745-2754

14. Plaha P, Gill SS. Bilateral deep brain stimulation of the pedunculopontine nucleus for Parkinson's disease. NeuroReport 2005; 16:1883-1887

15. Stefani A, Lozano AM, Peppe A, Stanzione P, Galati S, Tropepi D, et al. Bilateral deep brain stimulation of the pedunculopontine and subthalamic nuclei in severe Parkinson's disease. Brain 2007; 130:1596-1607

16. Khan S, Gill SS, Mooney L, White P, Whone A, Brooks DJ, et al. Combined pedunculopontine-subthalamic stimulation in Parkinson disease. Neurology 2012; 78:1090-1095

17. Ferraye MU, Debu B, Fraix V, White P, Whone A, Brooks DJ, et al. Effects of pedunculopontine nucleus area stimulation on gait disorders in Parkinson's disease. Brain 2010; 133:205-214

18. Moro E, Hamani C, Poon YY, Al-Khairallah T, Dostrovsky JO, Hutchison WD, et al. Unilateral pedunculopontine stimulation improves falls in Parkinson's disease. Brain 2010; 133:215-224

19. Thevathasan W, Coyne TJ, Hyam JA, Kerr G, Jenkinson N, Aziz TZ, et al. Pedunculopontine nucleus stimulation improves gait freezing in Parkinson disease. Neurosurgery 2011; 69:1248-1253 (discussion 1254)

20. Thevathasan W, Cole MH, Graepel CL, Hyam JA, Jenkinson N, Brittain JS, et al. A spatiotemporal analysis of gait freezing and the impact of pedunculopontine nucleus stimulation. Brain 2012; 135:1446-1454

21. Schaltenbrand G, Wahren W. Atlas for stereotaxy of the human brain. Stuttgart, New York: Thieme; 1977

22. Zrinzo L, Zrinzo LV, Tisch S, Limousin PD, Yousry TA, Afshar F, et al. Stereotactic localization of the human pedunculopontine nucleus : atlas based coordinates and validation of a magnetic resonance imaging protocol for direct localization. Brain, 2008. 131(Pt 6)1588-98.

23. Afshar F, Watkins ES, Yap JC. Stereotaxic atlas of the human brainstem and cerebellar nuclei. A variability study. New York: Raven Press; 1978 
24. Paxinos G, Huang XF. Atlas of the human brainstem. San Diego: Academic Press; 1995

25. Fahn S, Elton RL, and members of the UPDRS development committee. In: Fahn S, Marsden CD, Calne DB, Goldstein M, editors. Recent development in Parkinson's disease. Florham Park, NJ; 1987

26. Defer GL, Widner H, Marié RM, Rémy P, Levivier M. Core assessment program for surgical interventional therapies in Parkinson's disease (CAPSIT-PD). Mov Disord. $1999 ; 14: 572-84$

27. Schmidt R, Freidl W, Fazekas F, Reinhart B, Grieshofer P, Koch M, et al. The Mattis Dementia Rating Scale: normative data from 1,001 healthy volunteers. Neurology. 1994; 44(5):964-6

28. Langston JW, Widner H, Goetz CG, Brooks D, Fahn S, Freeman T, et al. Core assessment program for intracerebral transplantations (CAPIT). Mov Disord. 1992; $7(1): 2-13$

29. Montgomery SA, Asberg M. A new depression scale designed to be sensitive to change. Br J Psychiatry 1979; 134:382-9

30. Dujardin K, Sockeel P, Delliaux M, Destée A, Defebvre L. The Lille Apathy Rating Scale : validation of a caregiver-based version. Mov. Disord. 2008; 23(6):845-9.

31. Lozano AM, Gildenberg PL, Tasker RR. Textbook of Stereotactic and Functional Neurosurgery 2009

32. Talairach J, Tournoux P. Co-Planar Stereotaxic Atlas of the Human Brain, 3-D Proportional System: An Approach to Cerebral Imaging 1988 1st Edition 132 pp, 130

33. Lefranc M, Manto M, Merle P, Tir M, Montpellier D, Constant JM, et al. Targeting the Red Nucleus for Cerebellar Tremor. Cerebellum 2014; 13(3):372-7

34. Rouaud T, Drapier S, Raoul S, Rolland Y, Leray E, Verin M. Critères prédictifs radiologiques d'aggravation des signes axiaux après stimulation du noyau sousthalamique (NST) dans la maladie de Parkinson. Rev. Neurol. 2007; 165

35. Welter ML, Houeto JL, Tezenas du Montcel S, Mesnage V, Bonnet AM, Pillon B, et al. Clinical Predictive Factors of Subthalamic Stimulation in Parkinson's Disease. Brain 2002; 125:575-83 


\section{CORRELATION ENTRE FREEZING ET DIMENSION DU NOYAU PEDONCULO- PONTIN DANS LA MALADIE DE PARKINSON : ANALYSE SEMI- QUANTITATIVE BASEE SUR L'IRM CEREBRALE AVEC SEQUENCE FGATIR MODIFIEE}

Introduction : Les troubles de la marche constituent un problème majeur dans la maladie de Parkinson (MP). Le but de notre étude était de déterminer s'il existait une corrélation entre la taille du noyau pédonculo-pontin (PPN) et la présence d'un freezing.

Matériel et méthodes : Grâce à la séquence IRM Fast Gray Matter Acquisition T1 Inversion Recovery (FGATIR) modifiée, nous avons localisé le PPN et l'avons évalué de manière semiquantitative $(0=$ pas d'atrophie, $1=$ atrophie légère, $2=$ atrophie modérée, $3=$ atrophie sévère). Les patients avaient bénéficié de l'IRM dans le bilan pré-stimulation cérébrale profonde (SCP) des noyaux subthalamiques (NST). Les données cliniques et neuropsychologiques préopératoires ont été recueillies.

Résultats : 30 patients ont été inclus : 12 dans le groupe MP freezers (FOG +), 13 dans le groupe MP non freezers (FOG -) et 5 patients dans le groupe contrôle (patients dystoniques). Les coordonnées stéréotaxiques moyennes du PPN rostral étaient les suivantes : 6,58 mm en latéralité, 2,92 $\mathrm{mm}$ derrière la commissure postérieure $(\mathrm{CP})$ et $8,87 \mathrm{~mm}$ sous la ligne commissure antérieure - commissure postérieure (CA-CP), celles du PPN caudal : 7,18 mm en latéralité, 4,07 mm derrière $\mathrm{CP}$ et 13,18 mm sous la ligne CA-CP. Comparativement aux coordonnées recueillies par Zrinzo, la différence maximale était de 0,72 $\mathrm{mm}$. Dans le groupe FOG +, il existait davantage de PPN évalués à 2 ou 3 comparativement au groupe FOG (27\% versus $17 \%$ ). Toutefois, il n'existait pas de différence significative du degré d'atrophie du PPN, à droite ou à gauche.

Discussion : Si les tendances de nos résultats se confirment avec une population plus grande, la taille du PPN pourrait être évaluée en tant que facteur prédictif d'apparition de signes axiaux en postopératoire d'une SCP des NST.

Conclusion : La séquence FGATIR modifiée permet de réaliser une évaluation précise de la taille du PPN.

Mots clés : noyau pédonculo-pontin, maladie de Parkinson, freezing, IRM, SCP 


\section{RELATIONSHIP BETWEEN FREEZING AND PEDUNCULOPONTINE NUCLEUS' SIZE IN PARKINSON'S DISEASE: SEMI QUANTITATIVE ANALYSIS BASED ON MRI WITH MODIFIED FGATIR SEQUENCE}

Background: Gait disorders represent a major problem in Parkinson's disease (PD). The aim of this study was to determine the possible relation between pedunculopontine nucleus' size $(\mathrm{PPN})$ and occurrence of freezing.

Methods: Modified Fast Gray Matter Acquisition T1 Inversion Recovery images (FGATIR) was obtained using $3 \mathrm{~T}$ MRI during pre surgical procedure for subthalamic deep brain stimulation (STN-DBS) for Parkinson's disease (PD). We localized precisely PPN and evaluated it semi quantitatively $(0=$ no atrophy, $1=$ mild atrophy, $2=$ moderate atrophy, $3=$ severe atrophy). Clinical and neuropsychological data were collected.

Results: 30 patients were included: 12 in PD freezer group (FOG +), 13 in PD non freezer group (FOG -) and 5 in control group (dystonic patients). The mean stereotactic coordinates for the rostral PPN were $6.58 \mathrm{~mm}$ lateral, $2.92 \mathrm{~mm}$ posterior to the posterior commissure (PC) and $8.87 \mathrm{~mm}$ caudal to the anterior commissure - posterior commissure (AC-PC) plane, those of the caudal PPN were $7.18 \mathrm{~mm}$ lateral, $4.07 \mathrm{~mm}$ posterior to the PC and $13.18 \mathrm{~mm}$ caudal to the AC-PC. Comparatively to the coordinates of Zrinzo, the greatest difference observed was $0.72 \mathrm{~mm}$. In FOG + group, there were more PPN measured at 2 or 3 compared to FOG group (27\% versus 17\%). However degree of PPN atrophy was not significantly different between the three groups.

Discussion: Our results need to be confirmed by a larger study. Thus, a reduced size of PPN, could have a potential value as predictive factor of occurrence of axial signs in PD after STNDBS.

Conclusion: We highlight the great interest of modified FGATIR MRI image to evaluate precisely the size of the PPN.

Keywords: pedunculopontine nucleus, Parkinson's disease, freezing, MRI, DBS 